Published in final edited form as:

Nature. 2021 March ; 591(7851): 639-644. doi:10.1038/s41586-021-03207-w.

\title{
Evolution of antibody immunity to SARS-CoV-2
}

Christian Gaebler ${ }^{1,11}$, Zijun Wang ${ }^{1,11}$, Julio C. C. Lorenzi ${ }^{1,11}$, Frauke Muecksch ${ }^{2,11}$, Shlomo Finkin $^{1,11}$, Minami Tokuyama ${ }^{3,11}$, Alice Cho ${ }^{1,11}$, Mila Jankovic ${ }^{1,11}$, Dennis SchaeferBabajew $^{1,11}$, Thiago Y. Oliveira ${ }^{1,11}$, Melissa Cipolla ${ }^{1,11}$, Charlotte Viant ${ }^{1}$, Christopher $O$. Barnes $^{4}$, Yaron Bram ${ }^{5}$, Gaëlle Breton ${ }^{1}$, Thomas Hägglöf ${ }^{1}$, Pilar Mendoza1 ${ }^{1}$, Arlene Hurley ${ }^{6}$, Martina Turroja ${ }^{1}$, Kristie Gordon ${ }^{1}$, Katrina G. Millard ${ }^{1}$, Victor Ramos ${ }^{1}$, Fabian Schmidt ${ }^{2}$, Yiska Weisblum ${ }^{2}$, Divya $\mathrm{Jha}^{3}$, Michael Tankelevich ${ }^{3}$, Gustavo Martinez-Delgado ${ }^{3}, \mathrm{Jim} \mathrm{Yee}^{7}$, Roshni Patel ${ }^{1}$, Juan Dizon ${ }^{1}$, Cecille Unson-O'Brien ${ }^{1}$, Irina Shimeliovich ${ }^{1}$, Davide F. Robbiani $^{8}$, Zhen Zhao ${ }^{7}$, Anna Gazumyan ${ }^{1}$, Robert E. Schwartz ${ }^{5,9}$, Theodora Hatziioannou ${ }^{2}$, Pamela J. Bjorkman ${ }^{4}$, Saurabh Mehandru ${ }^{3, \varpi}$, Paul D. Bieniasz $^{2,10, \varpi}$, Marina Caskey $^{1, \bowtie}$, Michel C. Nussenzweig ${ }^{1,10, \bowtie}$

${ }^{1}$ Laboratory of Molecular Immunology, The Rockefeller University, New York, NY, USA. 2'Laboratory of Retrovirology, The Rockefeller University, New York, NY, USA. ${ }^{3}$ Division of Gastroenterology, Department of Medicine, Icahn School of Medicine at Mount Sinai, New York, NY, USA. ${ }^{4}$ Division of Biology and Biological Engineering, California Institute of Technology, Pasadena, CA, USA. ${ }^{5}$ Division of Gastroenterology and Hepatology, Department of Medicine, Weill Cornell Medicine, New York, NY, USA. ${ }^{6}$ Hospital Program Direction, The Rockefeller University, New York, NY, USA. ${ }^{7}$ Department of Pathology and Laboratory Medicine, Weill Cornell Medicine, New York, NY, USA. ${ }^{8}$ Institute for Research in Biomedicine, Università della Svizzera

under exclusive licence to Springer Nature Limited 2021

${ }^{\bowtie}$ Correspondence and requests for materials should be addressed to S.M., P.D.B., M. Caskey or M.C.N. saurabh.mehandru@mssm.edu; pbieniasz@ rockefeller.edu; mcaskey@ rockefeller.edu; nussen@ rockefeller.edu. Author contributions C.G., P.D.B., P.J.B., T. Hatziioannou, S.M. and M.C.N. conceived, designed and analysed the experiments. D.F.R., M. Caskey and C.G. designed clinical protocols. Z.W., J.C.C.L., F.M., S.F., M. Tokuyama, A.C., M.J., D.S.-B., F.S., Y.W., T. Hägglöf, P.M., G.B., C.V., C.O.B., K.G., D.J., J.Y., G.M.-D., Y.B., R.E.S. and Z.Z. carried out experiments. A.G. and M. Cipolla produced antibodies. A.H., D.S.-B., M. Turroja, K.G.M., M. Tankelevich, C.G. and M. Caskey recruited participants and executed clinical protocols. I.S., R.P, J.D. and C.U.-O. processed clinical samples. T.Y.O. and V.R. performed bioinformatic analysis. C.G, P.D.B., P.J.B., T. Hatziioannou, S.M. and M.C.N. wrote the manuscript with input from all co-authors.

Competing interests The Rockefeller University has filed a provisional patent application in connection with this work on which D.F.R. and M.C.N. are inventors (US patent 63/021,387). R.E.S. is on the scientific advisory board of Miromatrix Inc and is a consultant and speaker for Alnylam Inc. S.M. has served as a consultant for Takeda Pharmaceuticals, Morphic and Glaxo Smith Kline. Z.Z. received seed instruments and sponsored travel from ET Healthcare.

Reprints and permissions information is available at http://www.nature.com/reprints.

Data presentation

Extended Data Figures were arranged in Adobe Illustrator 2020.

Reporting summary

Further information on research design is available in the Nature Research Reporting Summary linked to this paper.

Code availability

Computer code to process the antibody sequences is available at GitHub (https://github.com/stratust/igpipeline).

Additional information

Supplementary information The online version contains supplementary material available at https://doi.org/10.1038/ s41586-021-03207-w.

Publisher's note Springer Nature remains neutral with regard to jurisdictional claims in published maps and institutional affiliations. Peer review information Nature thanks Michael Laue, Stanley Perlman and the other, anonymous, reviewer(s) for their contribution to the peer review of this work. 
Italiana, Bellinzona, Switzerland. ${ }^{9}$ Department of Physiology, Biophysics and Systems Biology, Weill Cornell Medicine, New York, NY, USA. ${ }^{10}$ Howard Hughes Medical Institute, The Rockefeller University, New York, NY, USA. ${ }^{11}$ These authors contributed equally: Christian Gaebler, Zijun Wang, Julio C. C. Lorenzi, Frauke Muecksch, Shlomo Finkin, Minami Tokuyama, Alice Cho, Mila Jankovic, Dennis Schaefer-Babajew, Thiago Y. Oliveira, Melissa Cipolla.

\section{Abstract}

Severe acute respiratory syndrome coronavirus 2 (SARS-CoV-2) has infected 78 million individuals and is responsible for over 1.7 million deaths to date. Infection is associated with the development of variable levels of antibodies with neutralizing activity, which can protect against infection in animal models ${ }^{1,2}$. Antibody levels decrease with time, but, to our knowledge, the nature and quality of the memory B cells that would be required to produce antibodies upon reinfection has not been examined. Here we report on the humoral memory response in a cohort of 87 individuals assessed at 1.3 and 6.2 months after infection with SARS-CoV-2. We find that titres of $\operatorname{IgM}$ and $\mathrm{IgG}$ antibodies against the receptor-binding domain (RBD) of the spike protein of SARS-CoV-2 decrease significantly over this time period, with IgA being less affected. Concurrently, neutralizing activity in plasma decreases by fivefold in pseudotype virus assays. By contrast, the number of RBD-specific memory B cells remains unchanged at 6.2 months after infection. Memory B cells display clonal turnover after 6.2 months, and the antibodies that they express have greater somatic hypermutation, resistance to RBD mutations and increased potency, indicative of continued evolution of the humoral response. Immunofluorescence and PCR analyses of intestinal biopsies obtained from asymptomatic individuals at 4 months after the onset of coronavirus disease 2019 (COVID-19) revealed the persistence of SARS-CoV-2 nucleic acids and immunoreactivity in the small bowel of 7 out of 14 individuals. We conclude that the memory B cell response to SARS-CoV-2 evolves between 1.3 and 6.2 months after infection in a manner that is consistent with antigen persistence.

Antibody responses to SARS-CoV-2 were initially characterized in a (6.2 months; range of 165 to 223 days) after the onset of symptoms. cohort of individuals convalescing from COVID-19 at approximately In this cohort, symptoms lasted for a median of 12 days (range of 0 to 40 days (1.3 months) after infection ${ }^{1}$. Between 31 August and 1644 days) during the acute phase, and $10(11 \%)$ of the participants were October 2020, 100 participants returned for a 6-month follow-up hospitalized. Consistent with other reports ${ }^{3,4}, 38$ (44\%) of the particistudy visit. Although the initial criteria allowed enrolment of the pants reported persistent long-term symptoms attributable to COVIDclose contacts of individuals with SARS-CoV-2 infection confirmed 19 (Methods, Supplementary Tables 1, 2). The duration and severity by reverse-transcription PCR (RT-PCR) ${ }^{1}, 13$ of the contacts did not of symptoms during acute disease was significantly greater among seroconvert and were excluded from further analyses. The remaining participants with persistent post-acute symptoms at the second study 87 participants with RT-PCR-confirmed SARS-CoV-2 infection and/ visit (Extended Data Fig. 1m-q). Importantly, all 87 participants tested or seroconversion returned for analysis at approximately 191 days negative for SARS-CoV-2 at the 6-month follow-up study visit using an approved saliva-based PCR assay (Methods). 
Participant demographics and clinical characteristics are shown in Supplementary Tables 1, 2.

\section{Plasma SARS-CoV-2 antibody reactivity}

Antibody reactivity in plasma to the RBD and nucleoprotein (N) of SARS-CoV-2 was measured by enzyme-linked immunosorbent assay (ELISA) and automated serological assays ${ }^{1,5,6}$. Anti-RBD assays were strongly correlated (anti-RBD IgG ELISA and PylonIgG, anti-RBD IgM ELISA and Pylon-IgM at 1.3 months, $r=0.9200$ and $r=0.7543, P<$ 0.0001, respectively) (Extended Data Fig. 2a-d), and anti-N assays showed a moderate correlation (anti-N IgG ELISA and Roche anti-N total immunoglobulin at 1.3 months, $r=$ 0.3596, $P=0.0012$ ) (Extended Data Fig. 2e, f). The anti-RBD and ELISA anti-N antibodies in plasma decreased significantly between 1.3 and 6.2 months (Fig. 1a-d). Notably, the decreased binding activity differed substantially by isotype and target. IgM showed the greatest decrease in anti-RBD reactivity (53\%), followed by $\operatorname{IgG}(32 \%)$; anti-RBD IgA decreased by only $15 \%$ and anti-N IgG levels by $22 \%$ (Fig. 1e). By contrast, the Roche anti$\mathrm{N}$ assay ${ }^{6}$ showed a small but significant increase (19\%) in reactivity between the two time points (Extended Data Fig. 2g), which might be explained by the use of an antigen bridging approach $^{7}$. In all cases, the magnitude of the decrease was inversely proportional to and directly correlated with the initial antibody levels, such that individuals with higher initial levels showed greater relative changes (Fig. 1f-i, Extended Data Fig. 2h). All measurements were strongly correlated between the two time points (Extended Data Fig. 2i-m) and anti-N IgG correlated with respective IgM, IgG and IgA anti-RBD reactivity at 1.3 months (Extended Data Fig. 2n-p). Notably, individuals with persistent post-acute symptoms had significantly higher levels of anti-RBD IgG and anti-N total antibody at both study visits (Extended Data Fig. 1a-1).

We measured plasma neutralizing activity using an HIV-1 virus pseudotyped with the SARS-CoV-2 spike protein ${ }^{1,8}$. Consistent with other reports ${ }^{9,10}$, the geometric mean halfmaximal neutralizing titre $\left(\mathrm{NT}_{50}\right)$ in this group of 87 participants was 401 and 78 at 1.3 and 6.2 months, respectively—representing a fivefold decrease (Fig. 1j, k). Neutralizing activity was directly correlated with the IgG anti-RBD ELISA measurements (Extended Data Fig. 2q, r). Moreover, the absolute magnitude of the decrease in neutralizing activity was inversely proportional to and directly correlated with the neutralizing activity at the earlier time point (Fig. 11). We conclude that antibodies to RBD and plasma neutralizing activity decrease significantly, but remain detectable, 6 months after infection with SARS-CoV-2 in the majority of individuals.

To examine the phenotypic landscape of circulating B cells, we performed high-dimensional flow cytometry on 41 randomly selected individuals at both time points and compared the results to pre-COVID-19 samples from healthy individuals $(n=20)$. Global highdimensional mapping with $t$-distributed stochastic neighbour embedding revealed significant persistent alterations in individuals who had recovered from COVID-19 (Extended Data Fig. 3a). The relative representation of clusters 2, 7,8 and 10 (corresponding to naive, memory, plasmablast and plasma cells, respectively) was decreased at 1.3 months and remained so at the later time point. Metacluster 15-which corresponds to immature B cells that are recent 
immigrants from the bone marrow-was increased at the early time point but returned to control levels at the end of the observation period (Extended Data Fig. 3b-d).

\section{SARS-CoV-2 memory B cell repertoire}

Whereas plasma cells are the source of circulating antibodies, memory B cells contribute to recall responses. To identify and enumerate the circulating SARS-CoV-2-specific memory B cell compartment, we used flow cytometry to isolate individual B lymphocytes with receptors that bound to $\mathrm{RBD}^{1}$ (Fig. 2a, Extended Data Fig. 4). Notably, the percentage of RBD-binding memory B cells increased marginally between 1.3 and 6.2 months in 21 randomly selected individuals (Fig. 2b).

To determine whether there were changes in the antibodies produced by memory B cells after 6.2 months, we obtained 532 paired antibody heavy and light chains from the same 6 individuals who were examined at the earlier time point ${ }^{1}$ (Supplementary Table 3 ). There was no significant difference in the representation of IGV genes at the two time points, including the over-representation of the IGHV3-30 and IGHV3-53 gene segments ${ }^{1,11-16}$ (Extended Data Fig. 5a, b). In keeping with this observation (and similar to the earlier time point), antibodies that shared the same IGHV and IGLV genes comprised $8.6 \%$ of all sequences in different individuals (Extended Data Fig. 5c). As might be expected, there was a small—but significant—overall increase in the percentage of IgG-expressing anti-RBD memory cells, from $49 \%$ to $58 \%(P=0.011)$ (Extended Data Fig. 5d-f). Consistent with the fractional increase in IgG memory cells, the extent of somatic hypermutation for both IGH and IGL differed significantly in all six individuals between the two time points. Whereas the average number of nucleotide mutations in IGH and IGL was only 4.2 and 2.8, respectively, at the first time point, these values were increased to 11.7 and 6.5, respectively, at the second time point $(P<0.0001)$ (Fig. 2c, Extended Data Fig. 6a-f). By contrast, the overall average length and hydrophobicity of complementarity-determining region 3 (CDR3) of IGH and IGL were unchanged (Extended Data Fig. 6g, h).

Similar to the earlier time point, we found expanded clones of memory B cells at 6.2 months (including 23 clones that appeared at both time points). However, expanded clones accounted for only $12.4 \%$ of all antibody sequences after 6.2 months, compared to $32 \%$ after 1.3 months $(P=0.0225)$ (Fig. 2d, e). In addition, the overall clonal composition of the memory compartment differed at the two time points in all of the individuals we examined (Fig. 2d). Forty-three expanded clones that were present at the earlier time point were not detectable after 6.2 months, and 22 new expanded clones appeared. In addition, the relative distribution of clones that appeared at both time points also varied. For example, the dominant clones in the individuals designated COV21 and COV57-in whom they represented $9.0 \%$ and $16.7 \%$ of all sequences, respectively, after 1.3 months - were reduced to $1.1 \%$ and $1.9 \%$, respectively, of all sequences after 6.2 months (Fig. 2d, Supplementary Table 3). We conclude that, although the magnitude of the RBD-specific memory B cell compartment is conserved between 1.3 and 6.2 months after infection with SARS-CoV-2, there is extensive clonal turnover and antibody sequence evolution that is consistent with prolonged germinal centre reactions. 


\section{SARS-CoV-2 monoclonal antibodies}

We tested 122 representative antibodies from the 6.2-month time point for reactivity to the RBD (Supplementary Table 4). The antibodies that we evaluated included: (1) 49 antibodies that were randomly selected from those antibodies that appeared only once; (2) 23 antibodies that appeared as singles at both 1.3 and 6.2 months; (3) 23 representatives of newly appearing expanded clones; and (4) 27 representatives of expanded clones appearing at both time points. One hundred and fifteen out of the 122 antibodies bound to RBD, which indicates that flow cytometry efficiently identified B cells that produce anti-RBD antibodies (Fig. 3a, Supplementary Tables 4, 5). Taking all antibodies together, the mean ELISA halfmaximal effective concentration $\left(\mathrm{EC}_{50}\right)$ was not significantly different at the two time points ${ }^{1}$ (Fig. 3a, Supplementary Table 4). However, comparison of the antibodies that were present at both time points revealed a significant improvement of the $\mathrm{EC}_{50}$ after 6.2 months $(P=0.0227)$ (Fig. 3b, Extended Data Fig. 7a).

To determine whether the antibodies expressed by memory B cells at the late time point also showed altered breadth, we compared them to earlier clonal relatives in binding assays using control and mutant RBDs. The substitutions E484K and Q493R ${ }^{17}$ were selected for resistance to class-2 antibodies (such as $\mathrm{C} 144$ and C121) that bind directly to the ACE2interaction ridge in the $\mathrm{RBD}^{1,18-20}$, and $\mathrm{R} 346 \mathrm{~S}, \mathrm{~N} 439 \mathrm{~K}$, and $\mathrm{N} 440 \mathrm{~K}$ were selected for resistance to class-3 antibodies (such as $\mathrm{C} 135$ ) that do not directly interfere with ACE2 binding ${ }^{1,17-20}$ (Fig. 3c). In addition, we also tested the V367F, A475V, S477N and V483A mutants of the RBD, which represent circulating variants that confer complete or partial resistance to class-1 and -2 antibodies ${ }^{17,18,21}$ (Fig. 3c). Out of 52 antibody clonal pairs that appeared at both time points, $43(83 \%)$ showed overall increased binding to mutant RBDs at the 6.2-month time point (Extended Data Fig. 7b-k, Supplementary Table 5). For example, C144 (an antibody recovered at the 1.3-month time point) was unable to bind to $\mathrm{RBD}(\mathrm{Q} 493 \mathrm{R})$ or $\mathrm{RBD}(\mathrm{E} 484 \mathrm{~K})$, but all five of its clonal derivatives collected at 6.2 months bound to $\operatorname{RBD}(\mathrm{Q} 493 \mathrm{R})$ and one also showed binding to $\mathrm{RBD}(\mathrm{E} 484 \mathrm{~K})$ (Fig. 3d). Overall, the most pronounced increase in binding occurred for mutations affecting the RBD in amino acid positions such as E484, Q493, N439, N440 and R346, which are critical for the binding of class-2 and -3 antibodies ${ }^{17,18}$ (Fig. 3e, Extended Data Fig. 7b-k, Supplementary Table $5)$.

Next, we tested all 122 antibodies from the 6.2-month time point for activity in a pseudotyped SARS-CoV-2 neutralization assay ${ }^{1,8}$ (Fig. 4a, Supplementary Table 6). Consistent with RBD-binding assays, the mean neutralization half-maximal inhibitory concentrations $\left(\mathrm{IC}_{50}\right)$ values were not significantly different at the two time points when all antibodies were compared $^{1}$ (Fig. 4a). However, comparison of the antibodies that were present at both time points revealed a significant improvement of the $\mathrm{IC}_{50}$ values at 6.2 months $(P=0.0003)$ (Fig. 4b, Extended Data Fig. 8a).

To determine whether the antibodies exhibiting altered RBD binding also show increased neutralizing breadth, we tested five representative antibody pairs recovered at the two time points against HIV-1 viruses pseudotyped with E484G, Q493R, and R346S mutant spike proteins (Fig. 4c, Supplementary Table 6). Notably, the Q493R and E484G pseudotyped 
viruses were resistant to neutralization by $\mathrm{C} 144$; by contrast, $\mathrm{C} 051$ (a 6.2 -month clonal derivative of C144) neutralized both variants, with $\mathrm{IC}_{50}$ values of 4.7 and $3.1 \mathrm{ng} \mathrm{ml}^{-1}$, respectively (Fig. 4c, d). Similarly, R346S pseudotyped viruses were resistant to C032, but $\mathrm{C} 080$ (a 6.2-month clonal derivative of C032) neutralized this variant with an $\mathrm{IC}_{50}$ of $5.3 \mathrm{ng}$ $\mathrm{ml}^{-1}$ (Fig. 4c, Extended Data Fig. 8b-f). Consistent with the observed changes in binding and neutralizing activity, several late-appearing antibodies (for example, C051) had acquired mutations directly in or adjacent to the RBD-binding paratope (Fig. 4e, Extended Data Fig. $8 \mathrm{~g}-\mathrm{j}$ ). We conclude that memory B cells that evolved during the observation period express antibodies with increased neutralizing potency and breadth.

\section{SARS-CoV-2 antigen persistence}

Antibody evolution occurs by somatic mutation and selection in germinal centres in which antigen can be retained in the form of immune complexes on the surface of follicular dendritic cells for prolonged periods of time. Residual protein in tissues represents another potential source of antigen. SARS-CoV-2 replicates in ACE2-expressing cells in the lungs, nasopharynx and small intestine ${ }^{22-25}$, and viral RNA has been detected in stool samples even after the virus is cleared from the nasopharyn $x^{26-28}$.

To determine whether there might be antigen persistence in the intestine after resolution of clinical illness, we obtained biopsies from the upper and lower gastrointestinal tract of 14 individuals at an average of 4 months (range of 2.8 to 5.7 months) after initial COVID-19 diagnosis (Supplementary Table 7). Immunostaining was performed to determine whether viral protein was also detectable in upper and lower gastrointestinal tract, with de-identified biopsies from individuals pre-dating the pandemic $(n=10)$ serving as controls. ACE2 and SARS-CoV-2 N protein was detected in intestinal enterocytes in 5 of 14 individuals (Fig. 5a-d, Extended Data Figs. 9a-h, 10a, b, Supplementary Table 7) but not in control samples (Extended Data Fig. 9i-1). When detected, immunostaining was sporadic, patchy, exclusive to the intestinal epithelium and not associated with inflammatory infiltrates (Extended Data Figs. 9a-h, 10a, b). Clinically approved nasopharyngeal-swab PCR assays were negative in all 14 individuals at the time of biopsy. However, biopsy samples from 3 of the 14 participants produced PCR amplicons that were sequence-verified as SARS-CoV-2 (Methods, Supplementary Table 7). In addition, viral RNA was detected by in situ hybridization in biopsy samples from the two participants who were tested (Extended Data Fig. 10c, d) but not in control samples (Extended Data Fig. 10e). Sampling variability during the endoscopic procedure probably contributed to incomplete concordance between detection of viral RNA and protein assays.

Neutralizing antibodies to SARS-CoV-2 develop in most individuals after infection but decay with time $7,9,10,29-32$. These antibodies are effective in prevention and therapy in animal models and are likely to have a role in protection from reinfection in humans ${ }^{2}$. Although there is a significant decrease in plasma neutralizing activity between 1.3 and 6.2 months, antibody titres remain measurable in most individu-als ${ }^{9,10,29-36}$.

Neutralizing monoclonal antibodies obtained from individuals during the early convalescence period showed notably low levels of somatic mutations, which some previous 
reports have attributed to defects in germinal centre formation ${ }^{11,12,14,37-40}$. Our data indicate that the anti-SARS-CoV-2 memory B cell response evolves during the first six months after infection, with accumulation of immunoglobulin somatic mutations, and production of antibodies with increased neutralizing breadth and potency. Persistent antibody evolution occurs in germinal centres and requires that B cells are exposed to antigen trapped in the form of immune complexes on follicular dendritic cells ${ }^{41}$. This form of antigen can be longlived, because follicular dendritic cells do not internalize immune complexes. In addition, even small amounts of persistent viral antigen could fuel antibody evolution. The observation that SARS-CoV-2 mRNA and protein remains detectable in the small intestinal epithelium in some individuals at months after infection is consistent with the relative persistence of anti-RBD IgA antibodies and continued antibody evolution ${ }^{34-36}$.

Memory responses are responsible for protection from reinfection and are essential for effective vaccination. The observation that memory B cell responses do not decay after 6.2 months ${ }^{34,35,42}$, but instead continue to evolve, is strongly suggestive that individuals who are infected with SARS-CoV-2 could mount a rapid and effective response to the virus upon reexposure.

\section{Online content}

Any methods, additional references, Nature Research reporting summaries, source data, extended data, supplementary information, acknowledgements, peer review information; details of author contributions and competing interests; and statements of data and code availability are available at https://doi.org/10.1038/s41586-021-03207-w.

\section{Methods}

\section{Data reporting}

No statistical methods were used to predetermine sample size. The experiments were not randomized and the investigators were not blinded to allocation during experiments and outcome assessment.

\section{Study participants}

Previously enrolled study participants ${ }^{1}$ were asked to return for a 6-month follow-up visit at the Rockefeller University Hospital from 31 August through to 16 October 2020. Eligible participants were adults aged 18-76 years and were either diagnosed with SARS-CoV-2 infection by RT-PCR (cases) or were close contacts (for example, members of the same household, coworkers or members of same religious community) of someone who had been diagnosed with SARS-CoV-2 infection by RT-PCR (contacts). Close contacts without seroconversion against SARS-CoV-2 as assessed by serological assays (described in 'Highthroughput automated serology assays') were not included in the subsequent analysis. Most study participants were residents of the greater New York city tri-state region and were asked to return approximately six months after the time of onset of COVID-19 symptoms. Participants presented to the Rockefeller University Hospital for blood sample collection and were asked to recall the symptoms and severity of clinical presentation during the acute (first six weeks) and the convalescent (seven weeks until second study visit) phase of COVID-19, 
respectively. The severity of acute infection was assessed by the World Health Organization (WHO) 'Ordinal Clinical Progression/Improvement Scale' (https://www.who.int/ publications/i/item/covid-19-therapeutic-trial-synopsis). Shortness of breath was assessed through the modified Medical Research Council dyspnoea scale ${ }^{43}$. Participants who presented with persistent symptoms attributable to COVID-19 were identified on the basis of chronic shortness of breath or fatigue, deficit in athletic ability and/or three or more additional long-term symptoms such as persistent unexplained fevers, chest pain, new-onset cardiac sequalae, arthralgias, impairment of concentration or mental acuity, impairment of sense of smell or taste, neuropathy or cutaneous findings ${ }^{3,4}$. All participants at Rockefeller University provided written informed consent before participation in the study and the study was conducted in accordance with good clinical practice. Clinical data collection and management were carried out using the software iRIS by iMedRIS. The study was performed in compliance with all relevant ethical regulations and the protocol for studies with human participants was approved by the Institutional Review Board (IRB) of the Rockefeller University.

\section{Gastrointestinal biopsy cohort}

To determine whether SARS-CoV-2 can persist in the gastrointestinal tract, we recruited a cohort of 14 individuals with prior diagnosis of and recovery from COVID-19 illness. Eligible participants included adults, 18-76 years of age who were previously diagnosed with SARS-CoV-2 by RT-PCR or through a combination of clinical symptoms consistent with COVID-19 plus evidence of seroconversion, and presented to the gastroenterology clinics of Mount Sinai Hospital. Endoscopic procedures were performed for clinically indicated conditions as detailed in Supplementary Table 7. All participants were asymptomatic at the time of the endoscopic procedures and negative for SARS-CoV-2 by nasal-swab PCR (cycle threshold $\left(C_{\mathrm{t}}\right)$ cut-off $<38$ ).

The CLIA-certified laboratory of the Mount Sinai Health System validated the laboratorydeveloped nasopharyngeal-swab real-time RT-PCR test according to the New York State Department of Health Wadsworth Center validation procedure for SARS-CoV-2 ${ }^{44}$. Informed consent was obtained from all participants. The biopsy-related studies were approved by the Mount Sinai Ethics Committee/IRB (IRB 16-0583, 'The impact of viral infections and their treatment on gastrointestinal immune cells').

\section{SARS-CoV-2 saliva PCR test}

The SARS-CoV-2 PCR method for saliva samples was developed and its performance characteristics determined by the Rockefeller University Clinical Genomics Laboratory. This laboratory-developed test has been authorized by New York state under an emergency use authorization for use by authorized laboratories. Saliva was collected into guanidine thiocyanate buffer as previously described ${ }^{45}$. RNA was extracted using either a columnbased (Qiagen QIAmp DSP Viral RNA Mini Kit, 61904) or a magnetic-bead-based method as previously described ${ }^{46}$. Reverse-transcribed cDNA was amplified using primers and probes validated by the Centers for Disease Control and Prevention or by Columbia University Personalized Medicine Genomics Laboratory, respectively, and approved by the 
US Food and Drug Administration under the emergency use authorization. Viral RNA was considered detected if the $C_{\mathrm{t}}$ for two viral primer and probe combinations was $<40$.

\section{Blood sample processing and storage}

Peripheral blood mononuclear cells were obtained by gradient centrifugation and stored in liquid nitrogen in the presence of FCS and DMSO. Heparinized plasma and serum samples were aliquoted and stored at $-20^{\circ} \mathrm{C}$ or below. Before experiments, aliquots of plasma samples were heat-inactivated $\left(56^{\circ} \mathrm{C}\right.$ for $\left.1 \mathrm{~h}\right)$ and then stored at $4{ }^{\circ} \mathrm{C}$.

\section{High-throughput automated serology assays}

Plasma samples from 80 out of 87 participants were tested by high-throughput automated serology assays. The Roche Elecsys anti-SARS-CoV-2 assay was performed on Roche Cobas e411 (Roche Diagnostics). The Elecsys anti-SARS-CoV-2 assay uses a recombinant protein representing the $\mathrm{N}$ antigen for the determination of antibodies against SARS-CoV-2. This assay received emergency use authorization approval from the US Food and Drug Administration ${ }^{6}$. The Pylon COVID19 IgG and IgM assays were used to measure plasma $\mathrm{IgG}$ and IgM antibodies against SARS-CoV-2, respectively. Plasma samples were assayed on the Pylon 3D analyser (ET HealthCare) as previously described ${ }^{5}$. This assay was implemented clinically as a laboratory-developed test under New York State Department of Health regulations. In brief, the assay was performed using a unitized test strip containing wells with predispensed reagents. The COVID-19 reagent contains biotinylated recombinant versions of the SARS-CoV-2 S-protein RBD and trace amounts of $\mathrm{N}$ protein as antigens that bind IgG and IgM, respectively. The cut-off values for both Pylon assays were determined using the mean of non-COVID-19 samples plus 6 s.d. The results of a sample are reported in the form of a cut-off index or an index value, which were determined by the instrument readout of the test sample divided by instrument readout at cut-off.

\section{ELISAs}

ELISAs $^{47,48}$ to evaluate antibodies binding to SARS-CoV-2 N (Sino Biological 40588V08B), RBD and additional RBDs were performed by coating of high-binding 96-half-well plates (Corning 3690) with $50 \mu \mathrm{l}$ per well of a $1 \mu \mathrm{g} \mathrm{ml}^{-1}$ protein solution in phosphatebuffered saline (PBS) overnight at $4{ }^{\circ} \mathrm{C}$. Plates were washed 6 times with washing buffer ( $1 \times$ PBS with $0.05 \%$ Tween-20 (Sigma-Aldrich)) and incubated with $170 \mu \mathrm{l}$ per well blocking buffer (1× PBS with $2 \%$ BSA and $0.05 \%$ Tween-20 (Sigma)) for $1 \mathrm{~h}$ at room temperature. Immediately after blocking, monoclonal antibodies or plasma samples were added in PBS and incubated for $1 \mathrm{~h}$ at room temperature. Plasma samples were assayed at a 1:67 starting dilution and 7 additional threefold serial dilutions. Monoclonal antibodies were tested at 10 $\mu \mathrm{g} \mathrm{ml}^{-1}$ starting concentration and 10 additional fourfold serial dilutions. Plates were washed 6 times with washing buffer and then incubated with anti-human $\operatorname{IgG}, \operatorname{IgM}$ or $\operatorname{IgA}$ secondary antibody conjugated to horseradish peroxidase (HRP) (Jackson Immuno Research 109-036-088 109-035-129 and Sigma A0295) in blocking buffer at a 1:5,000 dilution (IgM and $\operatorname{IgG}$ ) or 1:3,000 dilution (IgA). Plates were developed by addition of the HRP substrate, TMB (ThermoFisher) for $10 \mathrm{~min}$ (plasma samples) or $4 \mathrm{~min}$ (monoclonal antibodies), then the developing reaction was stopped by adding $50 \mu \mathrm{l} 1 \mathrm{M} \mathrm{H}_{2} \mathrm{SO}_{4}$ and absorbance was measured at $450 \mathrm{~nm}$ with an ELISA microplate reader (FluoStar Omega, BMG Labtech) 
with Omega and Omega MARS software for analysis. For plasma samples, a positive control (plasma from participant COV72, diluted 66.6-fold and 7 additional threefold serial dilutions in PBS) was added to every assay plate for validation. The average of its signal was used for normalization of all of the other values on the same plate with Excel software before calculating the AUC using Prism v.8.4 (GraphPad). For monoclonal antibodies, the $\mathrm{EC}_{50}$ was determined using four-parameter nonlinear regression (GraphPad Prism v.8.4).

\section{Expression of RBD proteins}

Mammalian expression vectors encoding the RBDs of SARS-CoV-2 (GenBank MN985325.1; S protein residues 319-539) and eight additional mutant RBD proteins (E484K, Q493R, R346S, N493K, N440K, V367F, A475V, S477N and V483A) with an Nterminal human IL-2 or Mu phosphatase signal peptide were previously described ${ }^{49}$.

\section{SARS-CoV-2 pseudotyped reporter virus}

SARS-CoV-2 pseudotyped particles were generated as previously described ${ }^{1,8}$. In brief, $293 \mathrm{~T}$ cells were transfected with pNL 4-3 $\Delta$ Env-nanoluc and pSARS-CoV-2-S ${ }_{\Delta 19}$. For generation of RBD-mutant pseudoviruses, pSARS-CoV-2-S ${ }_{\Delta 19}$ carrying either of the following spike mutations was used instead of its wild-type counterpart: Q493R, R346S or $\mathrm{E} 484 \mathrm{G}^{50}$. Particles were collected at $48 \mathrm{~h}$ after transfection, filtered and stored at $-80^{\circ} \mathrm{C}$.

\section{Pseudotyped virus neutralization assay}

Fourfold serially diluted plasma from individuals convalescent from COVID-19, or monoclonal antibodies, were incubated with SARS-CoV-2 pseudotyped virus for $1 \mathrm{~h}$ at 37 ${ }^{\circ} \mathrm{C}$. The mixture was subsequently incubated with $293 \mathrm{~T}_{\mathrm{ACE} 2}$ cells for $48 \mathrm{~h}$, after which cells were washed with PBS and lysed with Luciferase Cell Culture Lysis $5 \times$ reagent (Promega). Nanoluc luciferase activity in lysates was measured using the Nano- Glo Luciferase Assay System (Promega) with the Glomax Navigator (Promega). The obtained relative luminescence units were normalized to those derived from cells infected with SARS-CoV-2 pseudotyped virus in the absence of plasma or monoclonal antibodies. The half-maximal inhibitory concentration for plasma $\left(\mathrm{NT}_{50}\right)$ or monoclonal antibodies $\left(\mathrm{IC}_{50}\right)$ was determined using four-parameter nonlinear regression (least squares regression method without weighting; constraints: top $=1$, bottom $=0)($ GraphPad Prism $)$.

\section{High-dimensional data analysis of flow cytometry data}

High-dimensional viSNE and FlowSOM data analysis and visualization of flow cytometry data were performed on B cells using the Cytobank platform (https://cytobank.org). viSNE analysis was performed using equal sampling of 4,893 cells from each FCS file, with 75,00 iterations, a perplexity of 30 and a theta of 0.5 . The following markers were used to generate viSNE maps: IgA, CD305, TGFb-RII, CD138, CD10, CD272, IgD, CD24, CD21, CD95, HLA-DR, IgG, CD279, CD38, IgM, CD274, CD27, CD23, CXCR5, CD32, CD86, CD40, CD85j, CD11c and CXCR3. Resulting viSNE maps were fed into the FlowSOM clustering algorithm ${ }^{51}$. The self-organizing map was generated using hierarchical consensus clustering on the $t$-distributed stochastic neighbour embedding axes. 


\section{Heat map visualization}

Heat maps to display column-scaled $z$-scores of mean fluorescence intensity for individual FlowSOM clusters according to marker expression were created using the $\mathrm{R}$ function pheatmap.

\section{Biotinylation of viral protein for use in flow cytometry}

Purified and Avi-tagged SARS-CoV-2 RBD was biotinylated using the Biotin-Protein Ligase-BIRA kit according to manufacturer's instructions (Avidity) as previously described ${ }^{1}$. Ovalbumin (Sigma, A5503-1G) was biotinylated using the EZ-Link Sulfo-NHSLC-Biotinylation kit according to the manufacturer's instructions (Thermo Scientific). Biotinylated ovalbumin was conjugated to streptavidin-BV711 (BD biosciences, 563262) and RBD to streptavidin-PE (BD Biosciences, 554061) and streptavidin-AF647 (Biolegend, 405237) $)^{1}$.

\section{Single-cell sorting by flow cytometry}

Single-cell sorting by flow cytometry was previously described ${ }^{1}$. In brief, peripheral blood mononuclear cells were enriched for B cells by negative selection using a pan-B-cell isolation kit according to the manufacturer's instructions (Miltenyi Biotec, 130-101-638). The enriched B cells were incubated in FACS buffer ( $1 \times$ PBS, $2 \%$ FCS, 1 mM EDTA) with the following anti-human antibodies (all at 1:200 dilution): anti-CD20-PECy7 (BD Biosciences, 335793), anti-CD3-APC-eFluro 780 (Invitrogen, 47-0037-41), anti-CD8APC-eFluor 780 (Invitrogen, 47-0086-42), anti-CD16-APC-eFluor 780 (Invitrogen, 470168-41), anti-CD14-APC-eFluor 780 (Invitrogen, 47-0149-42), as well as Zombie NIR (BioLegend, 423105) and fluorophore-labelled RBD and ovalbumin (Ova) for $30 \mathrm{~min}$ on ice. Single CD3 ${ }^{-} \mathrm{CD} 8^{-} \mathrm{CD} 14^{-} \mathrm{CD} 16^{-} \mathrm{CD} 20^{+} \mathrm{Ova}^{-} \mathrm{RBD}-\mathrm{PE}^{+} \mathrm{RBD}-\mathrm{AF} 647^{+} \mathrm{B}$ cells were sorted into individual wells of 96 -well plates containing $4 \mu$ of lysis buffer $(0.5 \times \mathrm{PBS}, 10 \mathrm{mM}$ DTT, 3,000 units per ml RNasin ribonuclease inhibitors (Promega, N2615)) per well using a FACS Aria III and FACSDiva software (Becton Dickinson) for acquisition and FlowJo for analysis. The sorted cells were frozen on dry ice, and then stored at $-80^{\circ} \mathrm{C}$ or immediately used for subsequent RNA reverse transcription.

\section{Antibody sequencing, cloning and expression}

Antibodies were identified and sequenced as previously described ${ }^{1}$. In brief, RNA from single cells was reverse-transcribed (SuperScript III Reverse Transcriptase, Invitrogen, 18080-044) and the cDNA stored at $-20^{\circ} \mathrm{C}$ or used for subsequent amplification of the variable IGH, IGL and IGK genes by nested PCR and Sanger sequencing. Sequence analysis was performed using MacVector. Amplicons from the first PCR reaction were used as templates for sequence- and ligation-independent cloning into antibody expression vectors. Recombinant monoclonal antibodies and Fabs were produced and purified as previously described $^{1}$.

\section{Computational analyses of antibody sequences}

Antibody sequences were trimmed on the basis of quality and annotated using Igblastn v.1.14. with IMGT domain delineation system. Annotation was performed systematically 
using Change-O toolkit v.0.4.540 ${ }^{52}$. Heavy and light chains derived from the same cell were paired, and clonotypes were assigned on the basis of their $\mathrm{V}$ and $\mathrm{J}$ genes using in-house $\mathrm{R}$ and Perl scripts (Extended Data Fig. 5). All scripts and the data used to process antibody sequences are publicly available on GitHub (https://github.com/stratust/igpipeline).

The frequency distributions of human $\mathrm{V}$ genes in anti-SARS-CoV-2 antibodies from this study were compared to $131,284,220$ previously generated $\operatorname{IgH}$ and IgL sequences ${ }^{53}$ and downloaded from cAb-Rep ${ }^{54}$ (a database of human shared BCR clonotypes available at https://cab-rep.c2b2.columbia.edu/). On the basis of the 82 distinct $\mathrm{V}$ genes that make up the 1,703 analysed sequences from immunoglobulin repertoire of the three participants present in this study, we selected the IgH and IgL sequences from the database that are partially coded by the same $\mathrm{V}$ genes and counted them according to the constant region. The frequencies shown in (Extended Data Fig. 5) are relative to the source and isotype analysed. We used the two-sided binomial test to check whether the number of sequences belonging to a specific IGHV or IGLV gene in the repertoire is different according to the frequency of the same IGV gene in the database. Adjusted $P$ values were calculated using the false-discovery rate correction. In Extended Data Figs. 5, 6, significant differences are denoted with asterisks $(* P<0.05, * * P<0.01, * * * P<0.001, * * * * P<0.0001)$.

Nucleotide somatic hypermutation and CDR3 length were determined using in-house R and Perl scripts. For somatic hypermutations, IGHV and IGLV nucleotide sequences were aligned against their closest germlines using Igblastn and the number of differences were considered nucleotide mutations. The average mutations for $\mathrm{V}$ genes was calculated by dividing the sum of all nucleotide mutations across all participants by the number of sequences used for the analysis. To calculate the GRAVY scores of hydrophobicity ${ }^{55}$ we used the Guy H. R. Hydrophobicity scale based on free energy of transfer (kcal per mole $)^{56}$ implemented by the R package Peptides (https://CRAN.R-project.org/package=Peptides). We used 532 heavy-chain CDR3 amino acid sequences from this study and 22,654,256 IGH CDR3 sequences from the public database of memory B cell receptor sequences ${ }^{57}$. The Shapiro-Wilk test was used to determine whether the GRAVY scores were normally distributed. The GRAVY scores from all 532 IGH CDR3 amino acid sequences from this study were used to perform the test and 5,000 GRAVY scores of the sequences from the public database were randomly selected. The Shapiro-Wilk $P$ values were $6.896 \times 10^{-3}$ and $2.217 \times 10^{-6}$ for sequences from this study and the public database, respectively, indicating that the data were not normally distributed. Therefore, we used the two-tailed Wilcoxon nonparametric test to compare the samples, which indicated a difference in hydrophobicity distribution $\left(P=5 \times 10^{-6}\right)$ (Extended Data Fig. 6h).

A heat map of $\log _{2}$-transformed relative fold change in $\mathrm{EC}_{50}$ against the indicated $\mathrm{RBD}$ mutants for antibody clonal pairs obtained at 1.3 and 6.2 months (Fig. 3e, Extended Data Fig. 7k) was created with R pheatmap package (https://github.com/raivokolde/pheatmap) using Euclidean distance and Ward. 2 clustering method.

\section{Biopsies and immunofluorescence}

Endoscopically obtained mucosal biopsies were formalin-fixed and paraffin-embedded. Sections $(5 \mu \mathrm{m})$ were cut, dewaxed in xylene, and rehydrated in graded alcohol and PBS. 
Heat-induced epitope retrieval was performed in target retrieval solution (DAKO, S1699) using a commercial pressure cooker. Slides were then cooled to room temperature, washed in PBS and permeabilized for $30 \mathrm{~min}$ in $0.1 \%$ Triton X-100 in PBS. Nonspecific binding was blocked with $10 \%$ goat serum (Invitrogen, 50062Z) for $1 \mathrm{~h}$ at room temperature. Sections were then incubated with a combination of primary antibodies diluted in blocking solution overnight at $4{ }^{\circ} \mathrm{C}$. Slides were washed 3 times in PBS and then incubated in secondary antibody and DAPI $\left(1 \mu \mathrm{g} \mathrm{ml}^{-1}\right)$ for $1 \mathrm{~h}$ at room temperature. Sections were washed in PBS three times and then mounted with Fluoromount-G (Electron Microscopy Sciences, 1798425). Controls included omitting primary antibody (no primary 995 control) or substituting primary antibodies with nonreactive antibodies of the same isotype (isotype control). A Nikon Eclipse Ni microscope and digital SLR camera (Nikon, DS-Qi2) was used to visualize and image the tissue.

The antibody used to stain sections for $\mathrm{N}$ protein was raised in rabbits against SARS-CoV N and is cross-reactive with SARS-CoV-2 $\mathrm{N}$ protein $^{58}$ (Supplementary Table 8).

\section{SARS-CoV-2 PCR from intestinal biopsies}

To determine whether SARS-CoV-2 RNA is present in the gastrointestinal tract, we isolated RNA from endoscopically obtained mucosal biopsies using Direct-zol miniprep kit (Zymo research, R2050). Reverse-transcribed cDNA was amplified using 2019-nCov Ruo Kit (IDT) to detect viral nucleocapsid genomic RNA. Amplification of subgenomic nucleocapsid RNA was done using following primers and probe: sgLeadSARSCov2_F $5^{\prime}$ CGATCTCTTGTAGATCTGTTCTC-3'28, wtN_R4 5' -GGTGAACCAAGACGCAGTAT-3', wtN_P4 5'-/56-FAM/TAACCAGAA/ ZEN/TGGAGAACGCAGTGGG/3IABkFQ/-3' .

Quantitative PCR was performed using QuantTect probe PCR kit (Qiagen, 204345) under following conditions: $95^{\circ} \mathrm{C} 15 \mathrm{~s}, 95^{\circ} \mathrm{C} 15 \mathrm{~s}$ and $60^{\circ} \mathrm{C} 1 \mathrm{~min}$ using the Applied Biosystem QuantStudio 6 Flex Real-Time PCR System. Viral RNA was considered detected if the $C_{\mathrm{t}}$ for viral primer and probe combinations was $<40$. Samples from positive wells were column-purified and presence of N1 sequences additionally verified by Sanger sequencing.

\section{SARS-CoV-2 RNA detection by probe proximity ligation}

Probes were designed with a 20-25 nucleotide homology to SARS-CoV-2 genomic RNA. Probes were assessed by NCBI BLAST to exclude off target binding to other cellular transcripts. IDT OligoAnalyzer (Integrated DNA Technologies) was used to identify probe pairs with similar thermodynamic properties; melting temperature $45-60^{\circ} \mathrm{C}, \mathrm{GC}$ content of $40-55 \%$ and low self-complementary. The $3^{\prime}$ end of each one of the probes used for proximity ligation signal amplification is designed with a partially complementary sequence to the 61-bp-long backbone and partially to the 21-bp insert (Supplementary Table 8).

Single-molecule fluorescence in situ hybridization (smFISH) probes were designed with a complementary $3^{\prime}$ end to the biotin detection probe (Supplementary Table 8).

Paraffin-embedded samples were sectioned at $10 \mu \mathrm{m}$. Sections were deparaffinized using $100 \%$ xylene, $5 \mathrm{~min}$ at room temperature, repeated twice. Slides were rinsed in $100 \%$ ethanol, $1 \mathrm{~min}$ at room temperature, twice, and air-dried. Endogenous peroxidase activity was eliminated by treating the samples with $0.3 \%$ hydrogen peroxide, $10 \mathrm{~min}$ at room 
temperature followed by washing with DEPC-treated water. Samples were incubated $15 \mathrm{~min}$ at $95-100{ }^{\circ} \mathrm{C}$ in antigen retrieval solution (ACDBio) rinsed in DEPC-treated water and dehydrated in 100\% ethanol, 3 min at room temperature and air-dried. Tissue sections were permeabilized $30 \mathrm{~min}$ at $40{ }^{\circ} \mathrm{C}$ using RNAscope protease plus solution (ACDBio) and rinsed in DEPC-treated water.

Hybridization was performed overnight at $40^{\circ} \mathrm{C}$ in a buffer based on DEPC-treated water containing $2 \times$ SSC, $20 \%$ formamide (Thermo Fischer Scientific), $2.5 \%(\mathrm{v} / \mathrm{v})$

polyvinylsulfonic acid, $20 \mathrm{mM}$ ribonucleoside vanadyl complex (New England Biolabs), 40 $\mathrm{U} \mathrm{ml}^{-1} \mathrm{RNasin}$ (Promega), 0.1\% (v/v) Tween 20 (Sigma Aldrich), $100 \mu \mathrm{g} \mathrm{ml}^{-1}$ salmon sperm DNA (Thermo Fisher Scientific), $100 \mu \mathrm{g} \mathrm{ml}^{-1}$ yeast RNA (Thermo Fisher Scientific). DNA probes dissolved in DEPC-treated water were added at a final concentration of $100 \mathrm{nM}$ (Integrated DNA Technologies). Samples were washed briefly and incubated in a buffer containing $2 \times \mathrm{SSC}, 20 \%$ formamide, $40 \mathrm{U} \mathrm{ml}^{-1} \mathrm{RNasin}$ at $40{ }^{\circ} \mathrm{C}$ and then washed four times (5 min each) in wash buffer, PBS, $0.1 \%$ (v/v) Tween 20, and $4 \mathrm{U} \mathrm{ml}^{-1} \mathrm{RNasin}$ (Promega). Slides were then incubated with $100 \mathrm{nM}$ insert and backbone oligonucleotides in PBS, 1x SSC, $0.1 \%$ (v/v) Tween 20, $100 \mu \mathrm{g} \mathrm{ml}^{-1}$ salmon sperm DNA (Thermo Fisher Scientific), $100 \mu \mathrm{g} \mathrm{ml}^{-1}$ yeast RNA (Thermo Fisher Scientific), $40 \mathrm{U} \mathrm{ml}^{-1} \mathrm{RNasin}$ at $37^{\circ} \mathrm{C}$. After four washes, tissues were incubated at $37^{\circ} \mathrm{C}$ with $0.1 \mathrm{U} \mathrm{\mu l}^{-1} \mathrm{~T} 4 \mathrm{DNA}$ ligase (New England Biolabs) in $50 \mathrm{mM}$ Tris- $\mathrm{HCl}, 10 \mathrm{mM} \mathrm{MgCl} 2,1 \mathrm{mM}$ ATP, $1 \mathrm{mM}$ DTT, $250 \mu \mathrm{g} \mathrm{ml}^{-1}$ BSA,

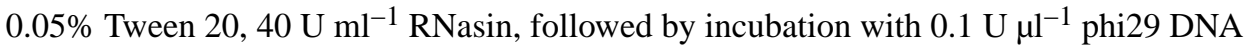
polymerase in $50 \mathrm{mM}$ Tris- $\mathrm{HCl}, 10 \mathrm{mM} \mathrm{MgCl} 2,10 \mathrm{mM}\left(\mathrm{NH}_{4}\right)_{2} \mathrm{SO}_{4}, 250 \mu \mathrm{M}$ dNTPs, $1 \mathrm{mM}$ DTT, $0.05 \%$ Tween $20,40 \mathrm{U} \mathrm{ml}^{-1} \mathrm{RNasin} \mathrm{pH} 7.5$ at $30^{\circ} \mathrm{C}$. Slides were washed and endogenous biotin was blocked using Avidin/Biotin blocking kit (Vector Laboratories) according to the manufacturer's instructions. Rolling cycle amplicons were identified using a biotin-labelled DNA probe at a concentration of $5 \mathrm{nM}$ at $37^{\circ} \mathrm{C}$ in PBS, $1 \times \mathrm{SSC}, 0.1 \%$ Tween $20,100 \mu \mathrm{g} \mathrm{ml}^{-1}$ salmon sperm DNA, $100 \mu \mathrm{g} \mathrm{ml}^{-1}$ yeast RNA. After washing, samples were incubated with 1:100 diluted streptavidin-HRP (Thermo Fisher Scientific) in PBS, 60 min at room temperature followed by washing. Fluorescent labelling was accomplished using Alexa Fluor 647 Tyramide SuperBoostKit (Thermo Fischer Scientific) according to the manufacturer's instructions. Hoechst 33342 was used for nuclear counterstaining (Thermo Fischer Scientific) and samples were mounted in ProLong gold antifade (Thermo Fischer Scientific).

\section{SARS-CoV-2 RNA detection by smFISH}

Hybridization was performed overnight at $40{ }^{\circ} \mathrm{C}$ in a buffer based on DEPC-treated water containing $2 \times$ SSC, $20 \%$ formamide (Thermo Fischer Scientific), $2.5 \%$ (v/v) polyvinylsulfonic acid, $20 \mathrm{mM}$ ribonucleoside vanadyl complex (New England Biolabs), 40 $\mathrm{U} \mathrm{ml}^{-1} \mathrm{RNasin}$ (Promega), 0.1\% (v/v) Tween 20 (Sigma Aldrich), $100 \mu \mathrm{g} \mathrm{ml}^{-1}$ salmon sperm DNA (Thermo Fisher Scientific), $100 \mu \mathrm{g} \mathrm{ml}^{-1}$ yeast RNA (Thermo Fisher Scientific). DNA probes dissolved in DEPC-treated water were added at a final concentration of $10 \mathrm{nM}$ (Integrated DNA Technologies). Samples were washed briefly and incubated in a buffer containing $2 \times \mathrm{SSC}, 20 \%$ formamide, $40 \mathrm{U} \mathrm{ml}^{-1} \mathrm{RNasin}$ at $40{ }^{\circ} \mathrm{C}$ and then washed four times in wash buffer, PBS, $0.1 \%$ (v/v) Tween 20, and $4 \mathrm{U} \mathrm{ml}^{-1} \mathrm{RNasin}$ (Promega). Samples were washed and endogenous biotin was blocked using Avidin/Biotin blocking kit (Vector 
Laboratories) according to the manufacturer's instructions. Slides were incubated with a biotin-labelled DNA probe at a concentration of $10 \mathrm{nM}$ at $37^{\circ} \mathrm{C}$ in PBS, $1 \times \mathrm{SSC}, 0.1 \%$ Tween 20, $100 \mu \mathrm{g} \mathrm{ml}^{-1}$ salmon sperm DNA, $100 \mu \mathrm{g} \mathrm{ml}^{-1}$ yeast RNA. After washing, samples were incubated with 1:100 diluted streptavidin-HRP (Thermo Fisher Scientific) in PBS, $60 \mathrm{~min}$ at room temperature followed by washing. Samples were labelled using ImmPACT-DAB substrate, counterstained using haematoxylin QS and imbedded in VectaMount AQ mounting medium (Vector Laboratories) according to the manufacturer's instructions.

\section{Extended Data}
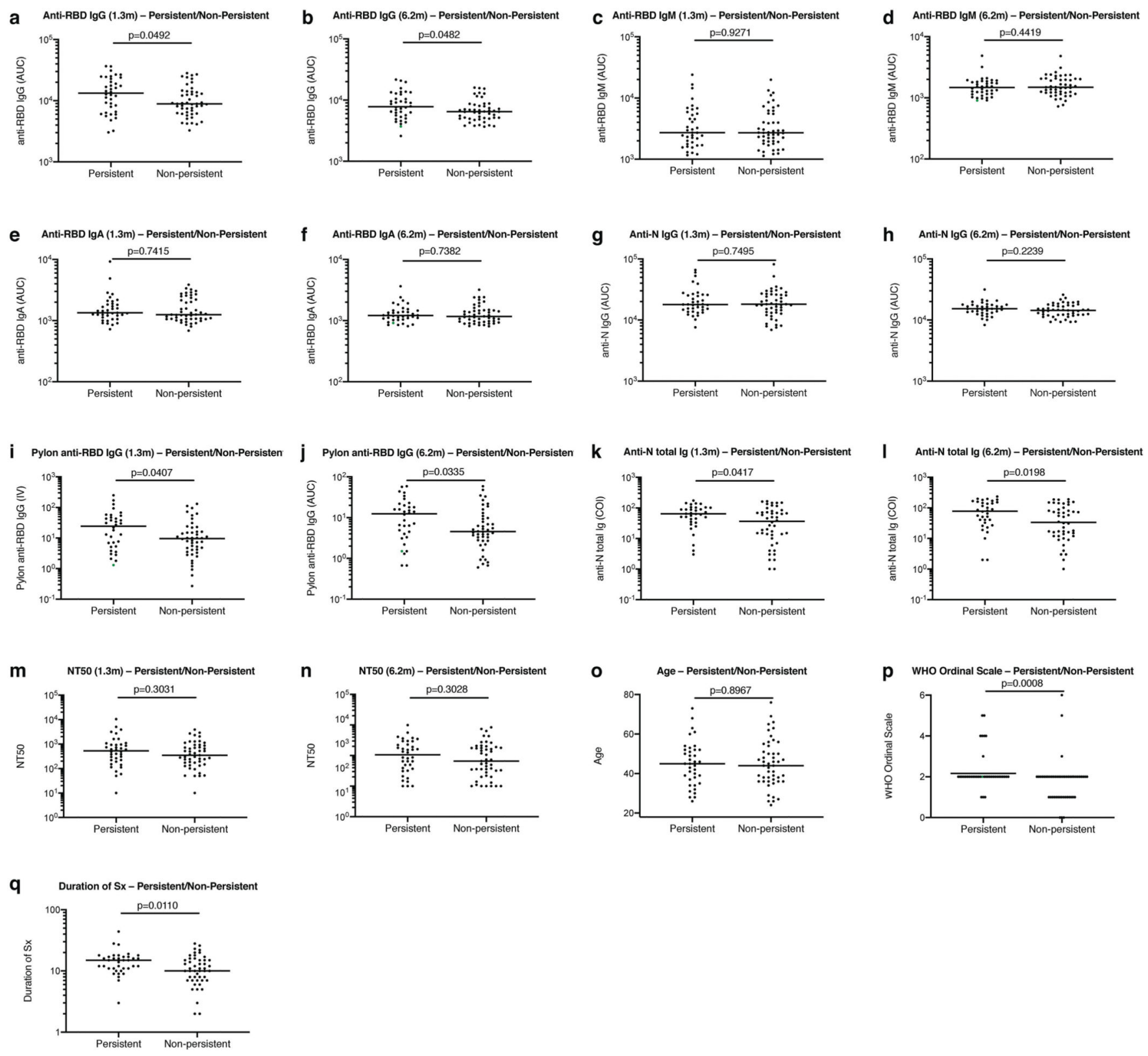

Extended Data Fig. 1 |. Clinical correlates of plasma antibody titres. 
a, Normalized AUC anti-RBD IgG titres at 1.3 months for participants with $(n=38)$ or without $(n=49)$ persistent post-acute symptoms. b, Normalized AUC anti-RBD IgG titres at 6.2 months for participants with $(n=38)$ or without $(n=49)$ persistent post-acute symptoms. c, Normalized AUC anti-RBD IgM titres at 1.3 months for participants with ( $n=$ 38 ) or without $(n=49)$ persistent post-acute symptoms. d, Normalized AUC anti-RBD IgM titres at 6.2 months for participants with $(n=38)$ or without $(n=49)$ persistent post-acute symptoms. e, Normalized AUC anti-RBD IgA titres at 1.3 months for participants with $(n=$ $38)$ or without $(n=49)$ persistent post-acute symptoms. f, Normalized AUC anti-RBD IgA titres at 6.2 months for participants with $(n=38)$ or without $(n=49)$ persistent post-acute symptoms. g, Normalized AUC anti-N IgG titres at 1.3 months for participants with $(n=38)$ or without $(n=49)$ persistent post-acute symptoms. h, Normalized AUC anti-N IgG titres at 6.2 months for participants with $(n=38)$ or without $(n=49)$ persistent post-acute symptoms. i, Index values (IV) of anti-RBD IgG titres at 1.3 months for participants with ( $n$ $=38)$ or without $(n=49)$ persistent post-acute symptoms. $\mathbf{j}$, Index values of anti-RBD IgG titres at 6.2 months for participants with $(n=38)$ or without $(n=49)$ persistent post-acute symptoms. k, Cut-off index (COI) values of anti-N total Ig titres at 1.3 months for participants with $(n=38)$ or without $(n=49)$ persistent post-acute symptoms. $\mathbf{1}$, COI values of anti-N total Ig titres at 6.2 months for participants with $(n=38)$ or without $(n=49)$ persistent post-acute symptoms. $\mathbf{m}, \mathrm{NT}_{50}$ values at 1.3 months for participants with $(n=38)$ or without $(n=49)$ persistent post-acute symptoms. $\mathbf{n}, \mathrm{NT}_{50}$ values at 6.2 months for participants with $(n=38)$ or without $(n=49)$ persistent post-acute symptoms. o, Age in years for participants with $(n=38)$ or without $(n=49)$ persistent post-acute symptoms. $\mathbf{p}$, Severity of acute infection as assessed by the WHO 'Ordinal Clinical Progression/ Improvement Scale' for participants with $(n=38)$ or without $(n=49)$ persistent post-acute symptoms. q, Duration of symptoms during acute infection for participants with $(n=38)$ or without $(n=49)$ persistent post-acute symptoms. Horizontal bars indicate median values. Statistical significance was determined using two-tailed Mann-Whitney $U$-tests. 

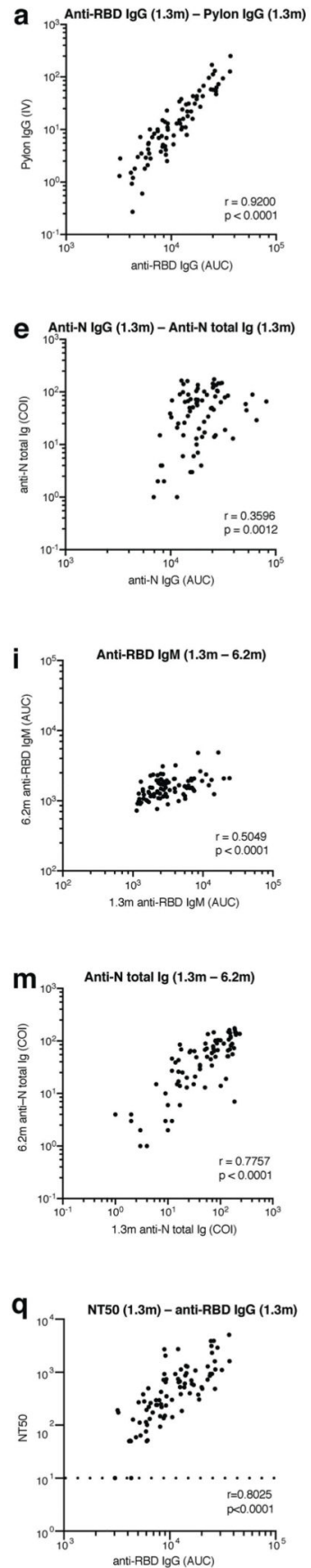
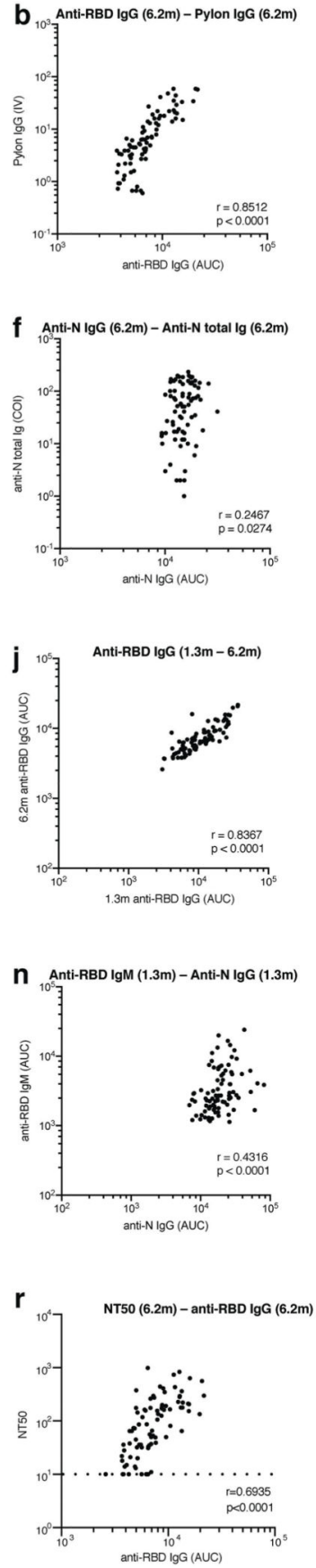
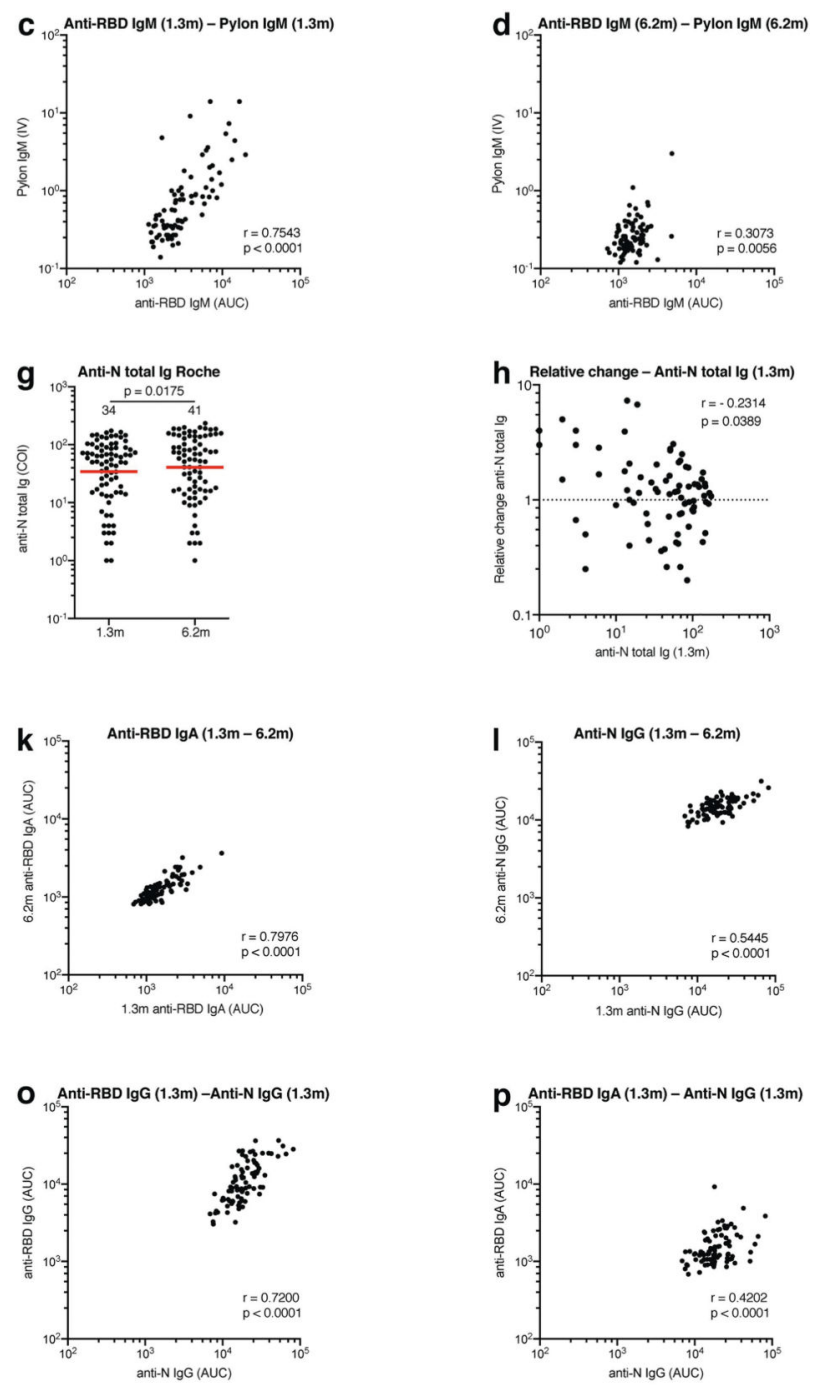

Extended Data Fig. 2 |. Correlations of plasma antibody measurements.

a, Normalized AUC for IgG anti-RBD plotted against Pylon IgG anti-RBD index values at 1.3 months. b, Normalized AUC for IgG anti-RBD plotted against Pylon IgG anti-RBD index values at 6.2 months. c, Normalized AUC for IgM anti-RBD plotted against Pylon IgM anti-RBD index values at 1.3 months. d, Normalized AUC for IgM anti-RBD plotted against Pylon IgM anti-RBD index values at 6.2 months. e, Normalized AUC for IgG anti-N plotted against Roche COI values for anti-N total Ig titres at 1.3 months. f, Normalized AUC for IgG anti-N plotted against Roche COI values for anti-N total Ig titres at 6.2 months. $\mathbf{g}$, 
Anti-N total Ig COI values for 80 individuals at the initial 1.3- and 6.2-month follow-up visit. $\mathbf{h}$, Relative change in anti-N total Ig levels between 1.3 and 6.2 months plotted against the anti-N total Ig levels at 1.3 months. i, Normalized AUC for IgM anti-RBD at 6.2 months plotted against IgM anti-RBD at 1.3 months. $\mathbf{j}$, Normalized AUC for IgG anti-RBD at 6.2 months plotted against IgG anti-RBD at 1.3 months. $\mathbf{k}$, Normalized AUC for IgA anti-RBD at 6.2 months plotted against IgA anti-RBD at 1.3 months. $\mathbf{l}$, Normalized AUC for IgG anti$\mathrm{N}$ at 6.2 months plotted against IgG anti-N at 1.3 months. $\mathbf{m}$, COI values for anti- $\mathrm{N}$ total Ig titres at 6.2 months plotted against anti-N total Ig titres at 1.3 months. $\mathbf{n}$, Anti-RBD IgM titres at 1.3 months plotted against anti-N IgG titres at 1.3 months. o, Anti-RBD IgG titres at 1.3 months plotted against anti-N IgG titres at 1.3 months. p, Anti-RBD IgA titres at 1.3 months plotted against anti-N IgG titres at 1.3 months. q, $\mathrm{NT}_{50}$ values at 1.3 months plotted against anti-RBD IgG titres at 1.3 months. $\mathbf{r}, \mathrm{NT}_{50}$ values at 6.2 months plotted against antiRBD IgG titres at 6.2 months. The $r$ and $P$ values were determined by two-tailed Spearman's correlations. 
a
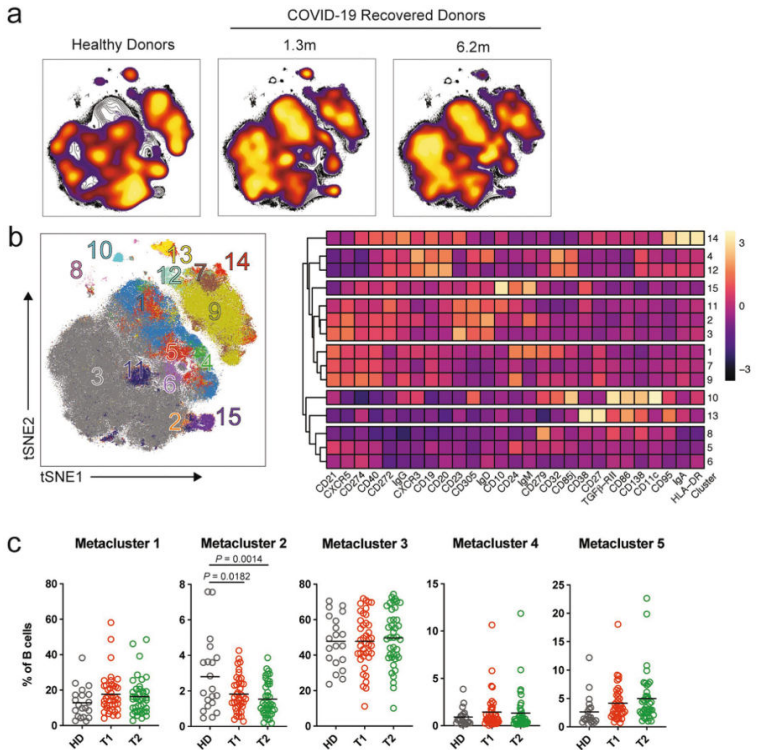

Metacluster 3 Metacluster 4 Metacluster 5
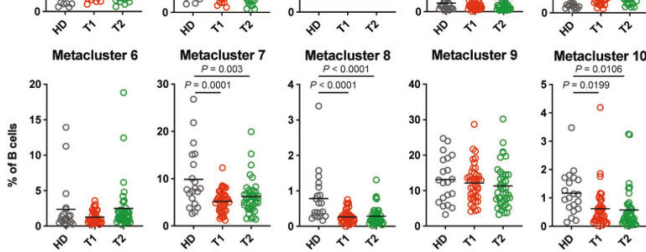

Metacluster 11 Metacluster 12 Metacluster 13 Metacluster 14 Metacluster 15
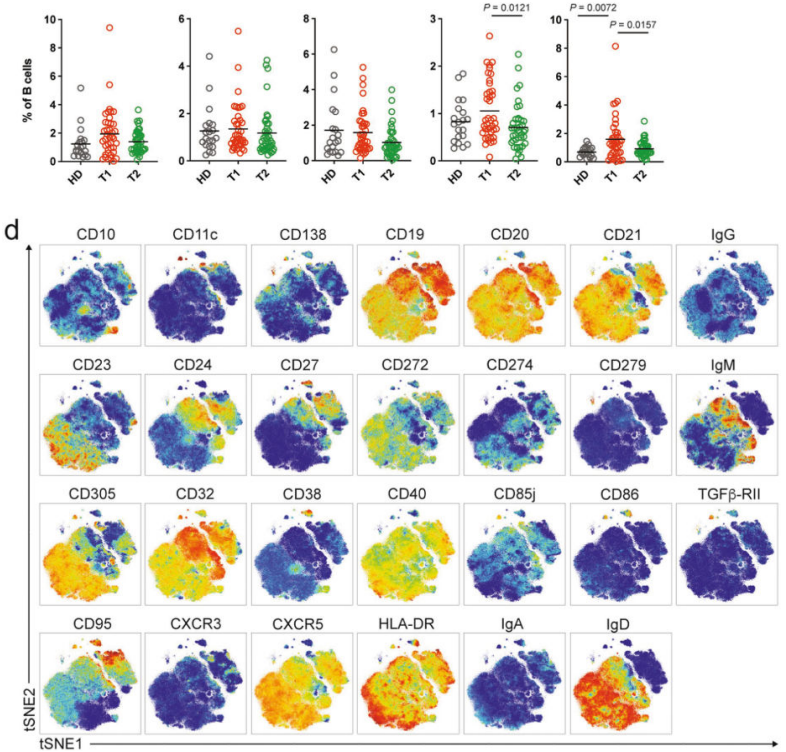

Extended Data Fig. 3 |. Persistent longitudinal changes in the phenotypic landscape of $B$ cells in individuals recovered from COVID-19.

a, Global viSNE projection of pooled B cells for all participants pooled shown in background contour plots, with overlaid projections of concatenated controls, and convalescent participants at 1.3 and 6.2 months. b, viSNE projection of pooled B cells for all participants of B cell clusters identified by FlowSOM clustering. Column-scaled $z$-scores of median fluorescence intensity (MFI) as indicated by cluster and marker. c, Frequency of B cells from each group in FlowSOM clusters indicated. Each circle represents an individual 
control individual $(n=20)$ (grey), convalescent participant at 1.3-month post-infection ( $n=$ $41)$ (red) or convalescent participant at 6.2 months post-infection $(n=41)$ (green).

Horizontal bars indicate mean values. Significance determined by two-tailed paired $t$-test for comparisons between time points within individuals and two-tailed unpaired $t$-test for comparison between controls and convalescent individuals. d, Individual viSNE projections of indicated protein expression.

a

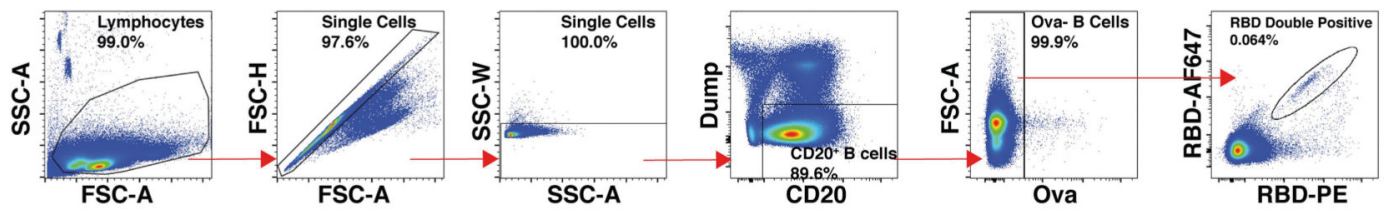

b
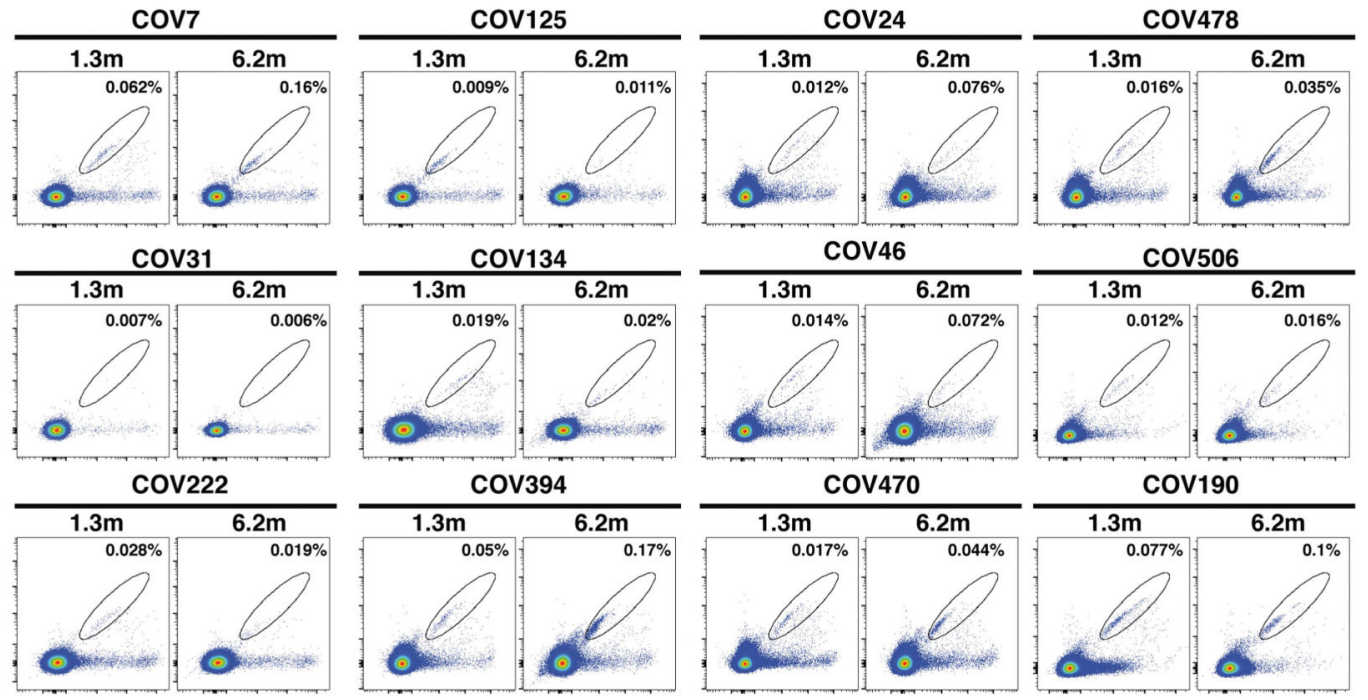

Cov287

cov149
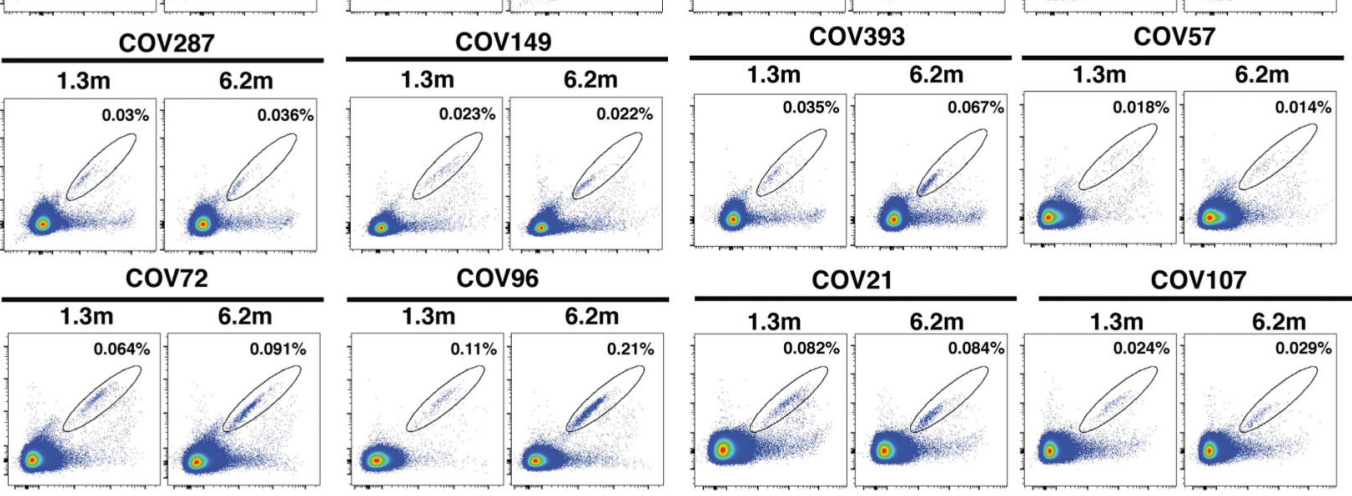

CoV107
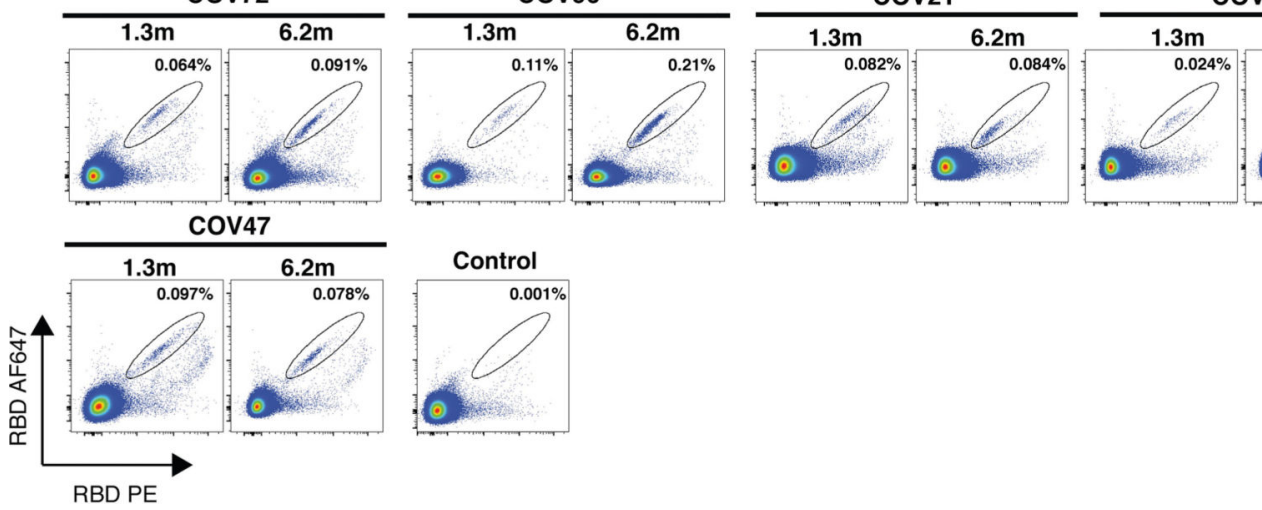

Extended Data Fig. 4 |. Flow cytometry.

Nature. Author manuscript; available in PMC 2021 June 23. 
a, Gating strategy used for cell sorting. Gating was on singlets that were $\mathrm{CD} 20^{+}$and $\mathrm{CD}^{-}{ }^{-} \mathrm{CD} 8{ }^{-} \mathrm{CD} 16^{-} \mathrm{Ova}^{-}$. Sorted cells were RBD-PE ${ }^{+}$and RBD-AF647 $7^{+}$. b, Flow cytometry showing the percentage of RBD-double-positive memory B cells from month 1.3 or month 6 post-infection in 21 randomly selected participants.
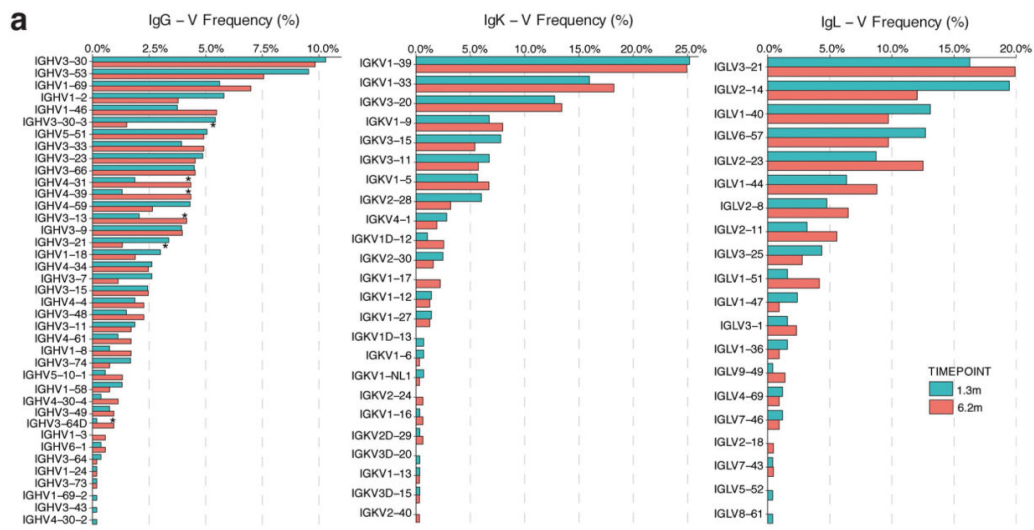

b

$\lg G$ - V Frequency $(\%)$
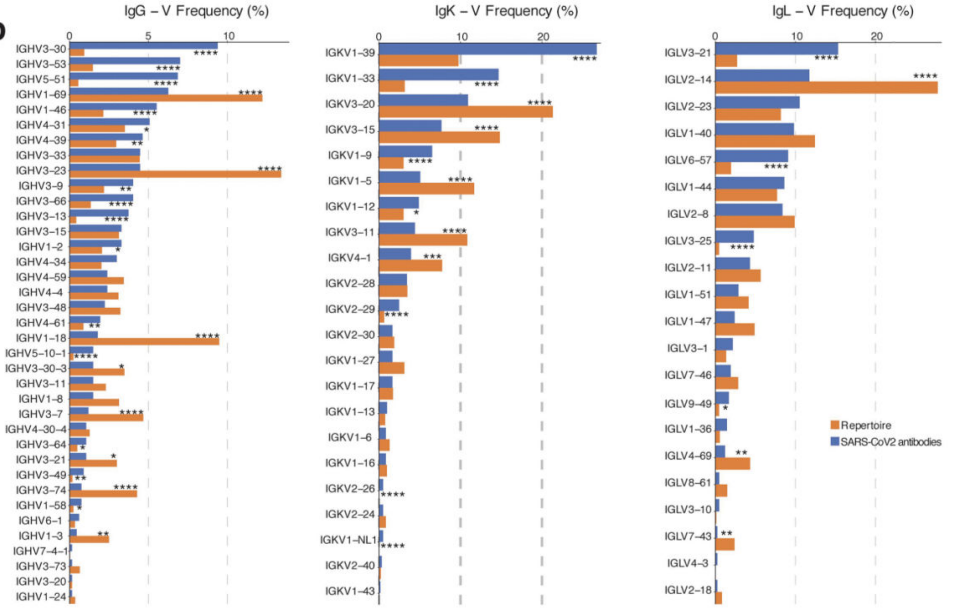

c

d
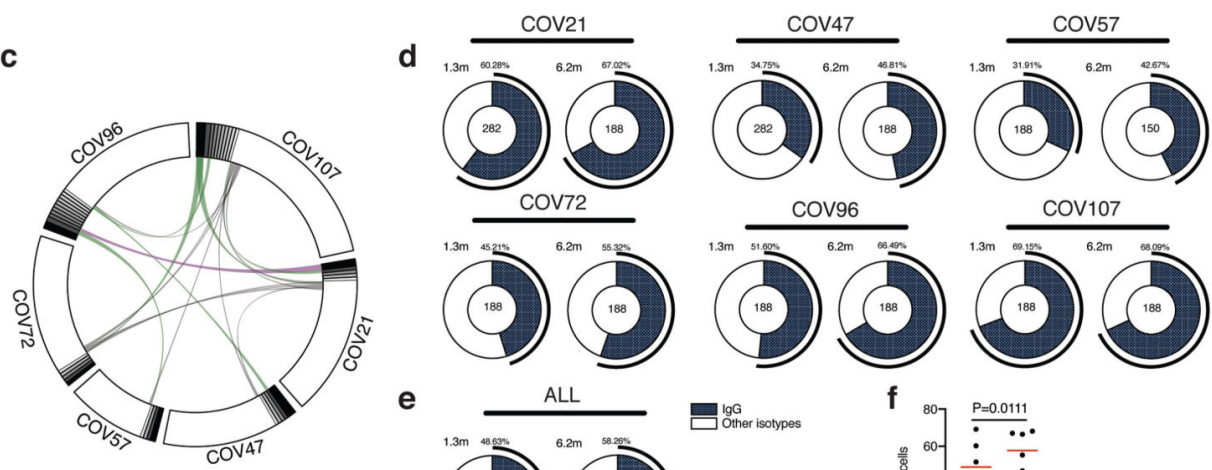

cov96
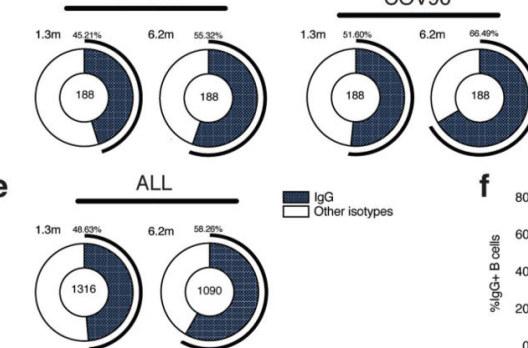

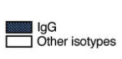

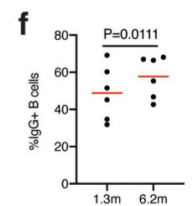

COV107

Extended Data Fig. 5 |. Frequency distributions of human $\mathbf{V}$ genes.

a, Two-sided binomial tests were used to compare the frequency distributions of human $\mathrm{V}$ genes of anti-SARS-CoV-2 antibodies from donors at 1.3 months and 6.2 months ${ }^{1} . * P<$ 
$0.05, * * P<0.01 * * * P<0.001, * * * * P<0.0001$. b, Two-sided binomial tests were used to compare the frequency distributions of human $\mathrm{V}$ genes of anti-SARS-CoV-2 antibodies from this study to sequences from ref. ${ }^{53}$. $* P<0.05, * * P<0.01, * * * P<0.001$, **** $P<0.0001$. c, Sequences from all six individuals with clonal relationships depicted as circos plots as in Fig. 2d. Interconnecting lines indicate the relationship between antibodies that share $\mathrm{V}$ and $\mathrm{J}$ gene-segment sequences at both IGH and IGL. Purple, green and grey lines connect related clones, clones and singles, and singles to each other, respectively. d, For each participant, the number of IgG heavy-chain sequences (black) analysed at month 1.3 (left) or month 6.2 post-infection (right). The number in the inner circle indicates the number of cells that was sorted for each individual denoted above the circle. e, The same as d but showing combined data for all six participants. f, Comparison of the percentage of IgG-positive B cells from all six individuals at month 1.3 or month 6.2 post-infection. The horizontal bars indicate the mean. Statistical significance was determined using two-tailed $t$-test. 
a

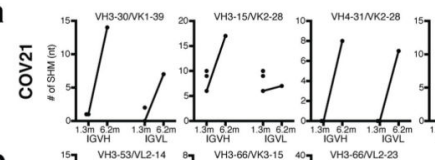

b

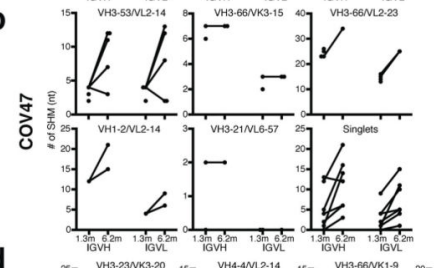

d

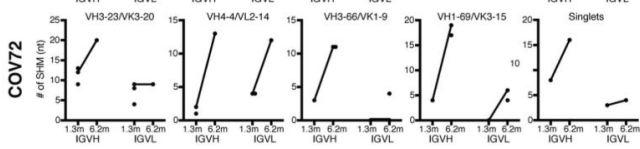

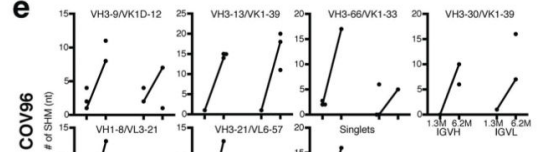

C ${ }^{15} 7$ VHS.51101-40
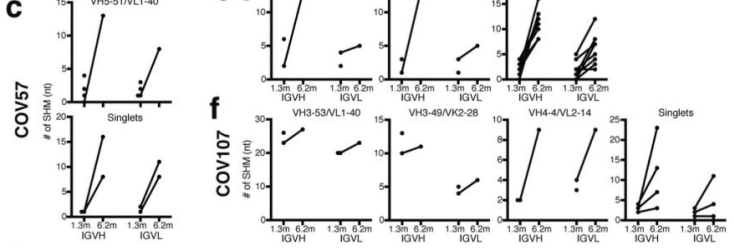

g
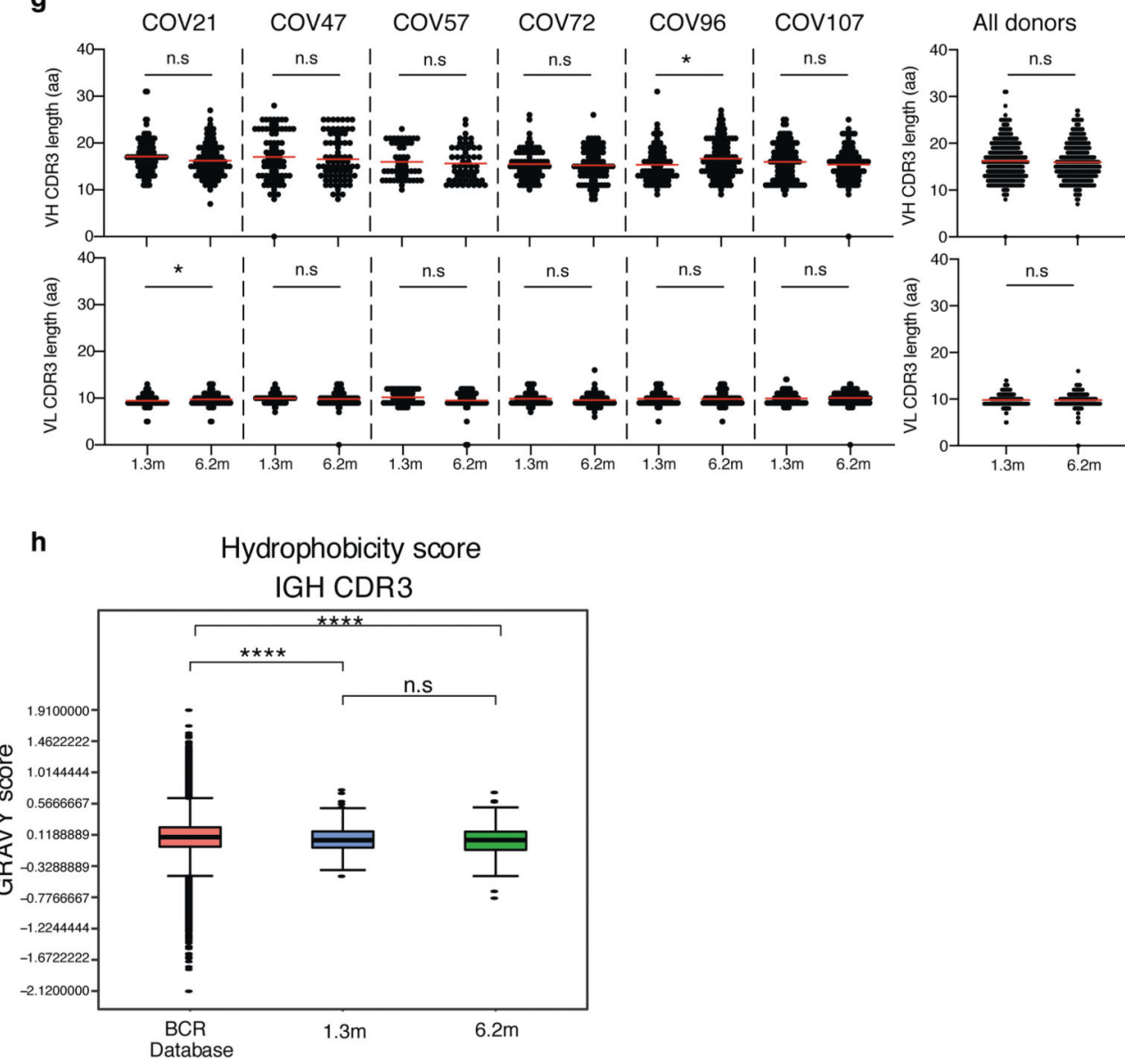

Extended Data Fig. 6 |. Analysis of antibody somatic hypermutation of persisting clones, CDR3 length and hydrophobicity.

a-f, Number of somatic nucleotide mutations in both the IGVH and IGVL of persisting clones found at month-1.3 and month-6.2 time points in six participants: COV21 (a), COV47 (b), COV57 (c), COV72 (d), COV96 (e) and COV107 (f). The VH and VL gene usage of each clonal expansion is indicated above the graphs, or are indicated as 'singlets' if the persisting sequence was isolated only once at both time points. Connecting line indicates the somatic hypermutation of the clonal pairs that were expressed as a recombinant 
monoclonal antibodies. g, For each individual, the amino acid length of the CDR3 at IGVH and IGVL is shown. The horizontal bars indicate the mean. The number of antibody sequences (IGVH and IGVL) evaluated for each participant are $n=90$ (COV21), $n=78$ (COV47), $n=53$ (COV57), $n=87$ (COV72), $n=104$ (COV96), $n=120$ (COV107). Right, all antibodies combined ( $n=532$ for both IGVH and IGVL). Statistical significance was determined using two-tailed Mann-Whitney $U$-tests and horizontal bars indicate median values. NS, not significant, $* P<0.05, * * P<0.01$, $* * * P<0.001$, $* * * * P<0.0001$. h, Distribution of the hydrophobicity GRAVY scores at the IGH CDR3 in 532 antibody sequences from this study compared to a public database: statistical analysis is provided in Methods. The box limits are at the lower and upper quartiles, the centre line indicates the median, the whiskers are $1.5 \times$ interquartile range and the dots represent outliers. Statistical significance was determined using two-tailed Wilcoxon matched-pairs signed-rank test. NS, not significant, $* P<0.05$, $* * P<0.01, * * * P<0.001$, $* * * * P<0.0001$ ). 


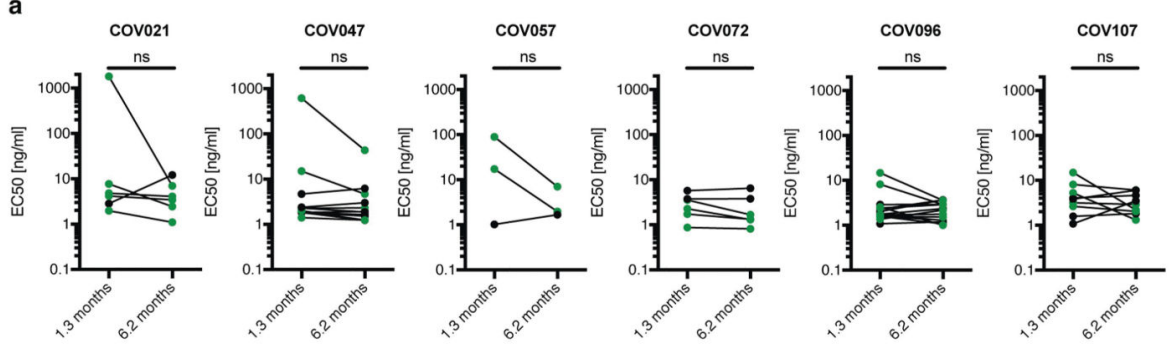

b

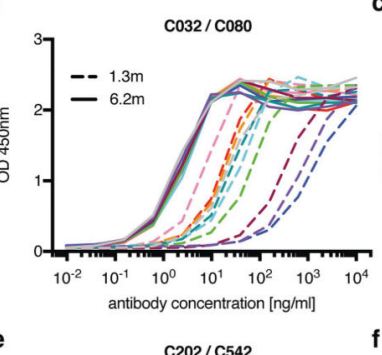

C

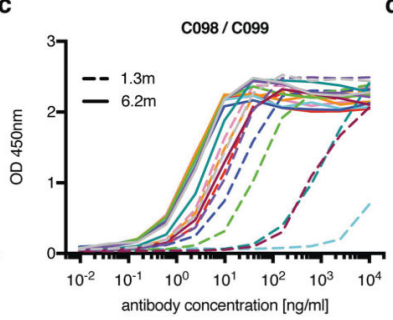

d
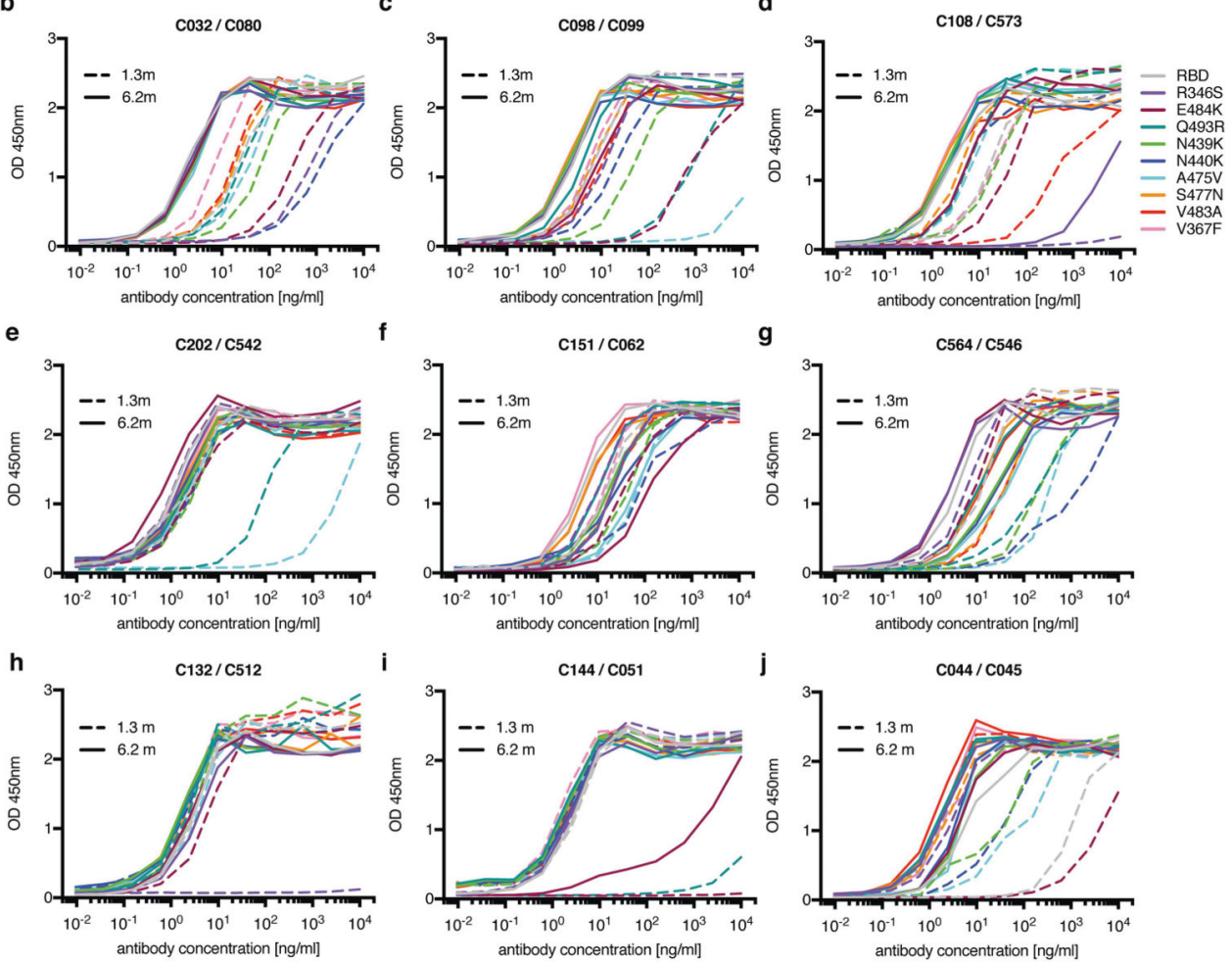

k

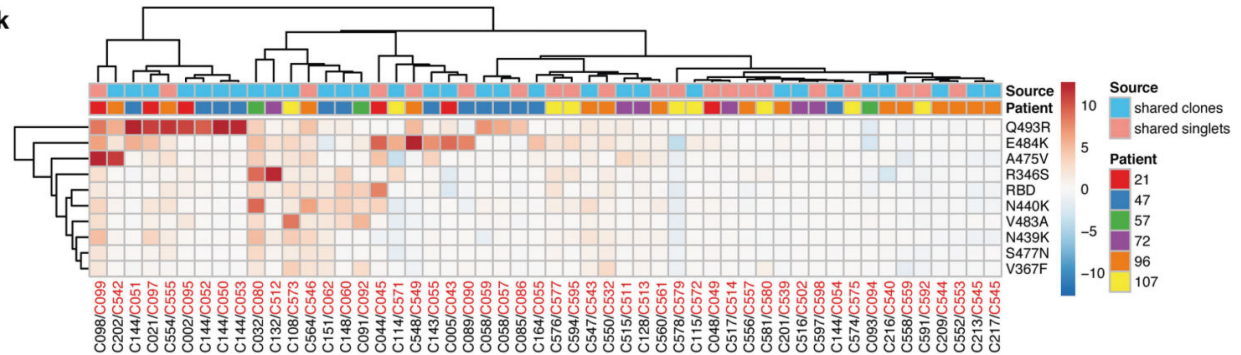

Extended Data Fig. 7 |. ELISA of wild-type or mutant RBD for monoclonal antibodies.

a, $\mathrm{EC}_{50}$ values for binding to wild-type RBD of shared singlets and shared clones of monoclonal antibodies obtained at the initial 1.3- and 6.2-month follow-up visit, divided by participant ( $n=6$ (COV21), $n=13$ (COV47), $n=3$ (COV57), $n=6$ (COV72), $n=15$ (COV96), $n=9$ (COV107)). Lines connect shared singlets or clones. Monoclonal antibodies with improved $\mathrm{EC}_{50}$ at the 6.2-month follow-up visit are highlighted in green; remaining monoclonal antibodies are shown in black. Statistical significance was determined using two-tailed Wilcoxon matched-pairs signed-rank test. b-j, Graphs show ELISA binding 
curves for different antibodies obtained at 1.3 months (dashed lines) and their clonal relatives obtained after 6.2 months (solid lines) binding to wild type, R346S, E484K, Q493R, N439K, N440K, A475V, S477N, V483A and V367F RBDs (colours as indicated). Antibody identifiers of pairs are as indicated on top of panels (1.3 months/6.2 months). $\mathbf{k}$, Heat map shows $\log _{2}$-transformed relative fold change in $\mathrm{EC}_{50}$ against the indicated RBD mutants for 52 antibody clonal pairs obtained at 1.3 (black) and 6.2 months (red). The clonal and participant origin for each antibody pair is indicated above.
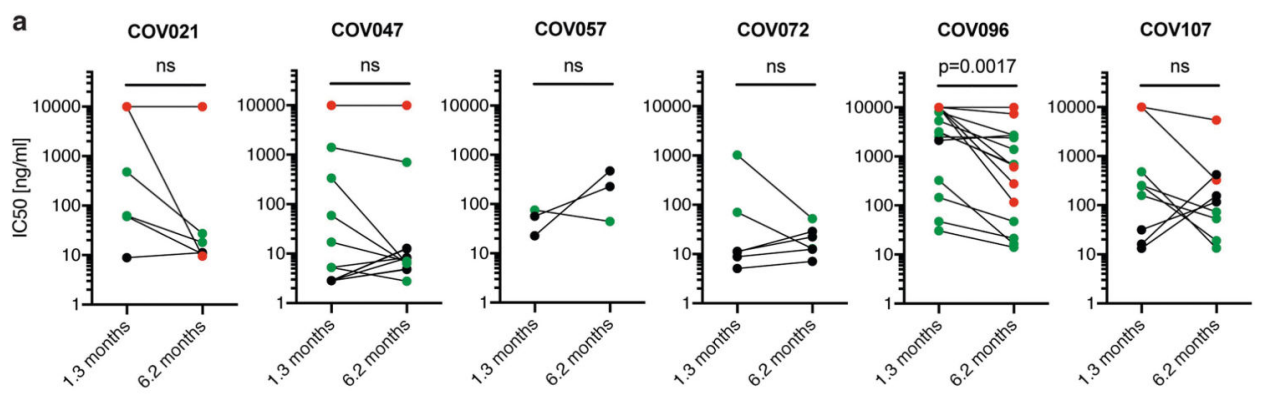

b
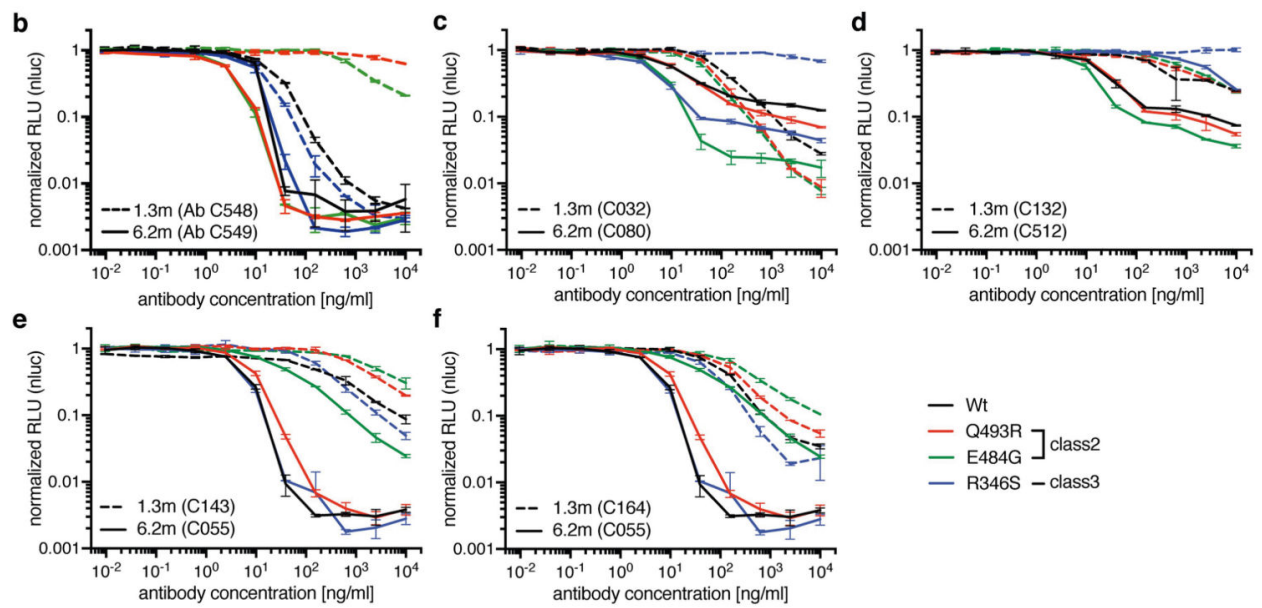

$$
\text { g }
$$

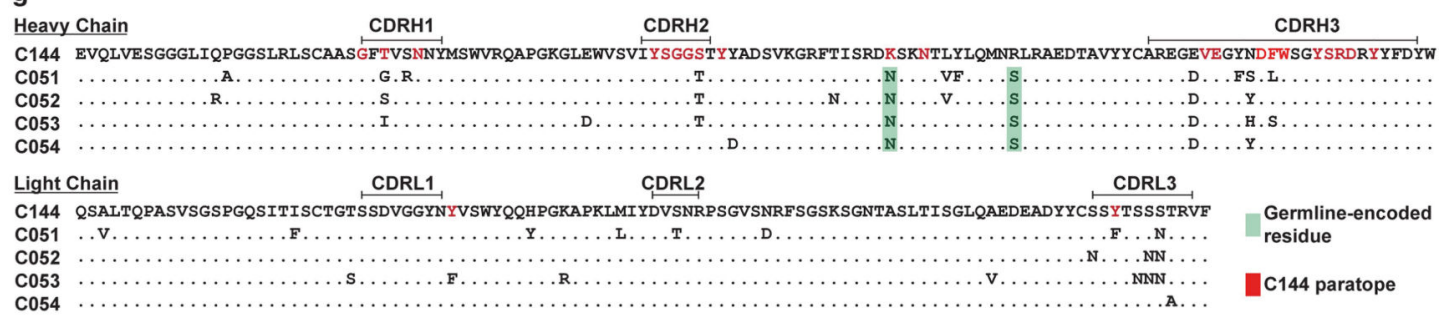
cos

h

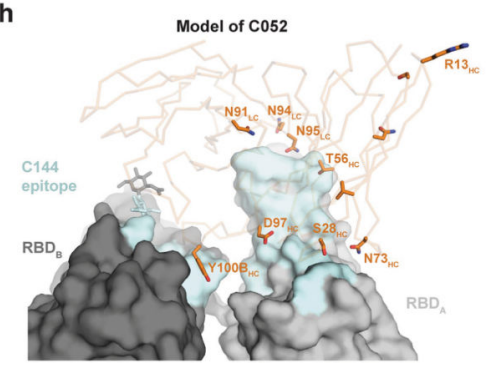

j

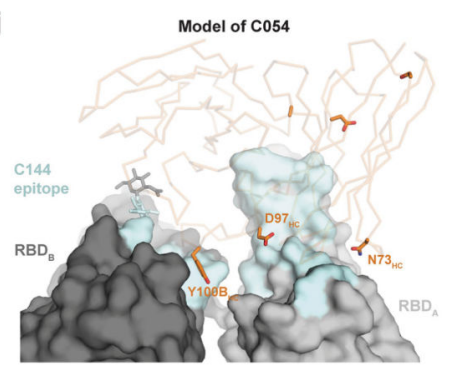

Nature. Author manuscript; available in PMC 2021 June 23. 
Extended Data Fig. 8 |. Neutralization of wild-type and mutant RBDs, C51 alignment and binding projection.

a, $\mathrm{IC}_{50}$ values of shared singlets and shared clones of monoclonal antibodies obtained at the initial 1.3- and 6.2-months follow-up visit, divided by participant ( $n=6$ (COV21), $n=13$ (COV47), $n=3$ (COV57), $n=6$ (COV72), $n=15$ (COV96), $n=9$ (COV107)). Lines connect shared singlets or clones. Monoclonal antibodies with undetectable $\mathrm{IC}_{50}$ at 1.3 months are plotted at $10 \mu \mathrm{g} \mathrm{ml}^{-1}$ and are highlighted in red; monoclobal antibodies with improved $\mathrm{IC}_{50}$ at the 6.2-month follow-up visit are highlighted in green; remaining monoclonal antibodies are shown in black. Statistical significance was determined using two-tailed Wilcoxon matched-pairs signed-rank test. b-f, The normalized relative luminescence values for cell lysates of $293 \mathrm{~T}_{\mathrm{ACE} 2}$ cells $48 \mathrm{~h}$ after infection with SARSCoV-2 pseudovirus containing wild-type RBD or RBD mutants (wild-type, Q493R, E484G and R346S RBD shown in black, red, green and blue, respectively) in the presence of increasing concentrations of monoclonal antibodies obtained at the 1.3-month initial visit $(1.3 \mathrm{~m}$, dashed lines) and their shared clones or singlets at the 6.2-month follow-up visit (6.2m, continuous lines). Antibody identifiers are as indicated. g, VH and VL amino acid sequence alignment of C144 and derivative antibodies C051, C052, C053 and C054. Germline-encoded residues are highlighted in green. Residues in the proximity of RBDbinding C144 paratope are highlighted in red. h-j, Surface representation of two adjacent 'down' RBDs $\left(\mathrm{RBD}_{\mathrm{A}}\right.$ and $\left.\mathrm{RBD}_{\mathrm{B}}\right)$ on a spike trimer with the $\mathrm{C} 144$ epitope on the RBDs highlighted in cyan and positions of amino acid mutations that accumulated in C052 (h), C053 (i) and C054 (j), compared to the parent antibody C144, highlighted as stick side chains on a $\mathrm{Ca}$ atom representation. The C052, C053 and C054 interactions with two RBDs was modelled on the basis of a cryo-electron microscopy structure of C144 Fab bound to spike trimer ${ }^{18}$. 

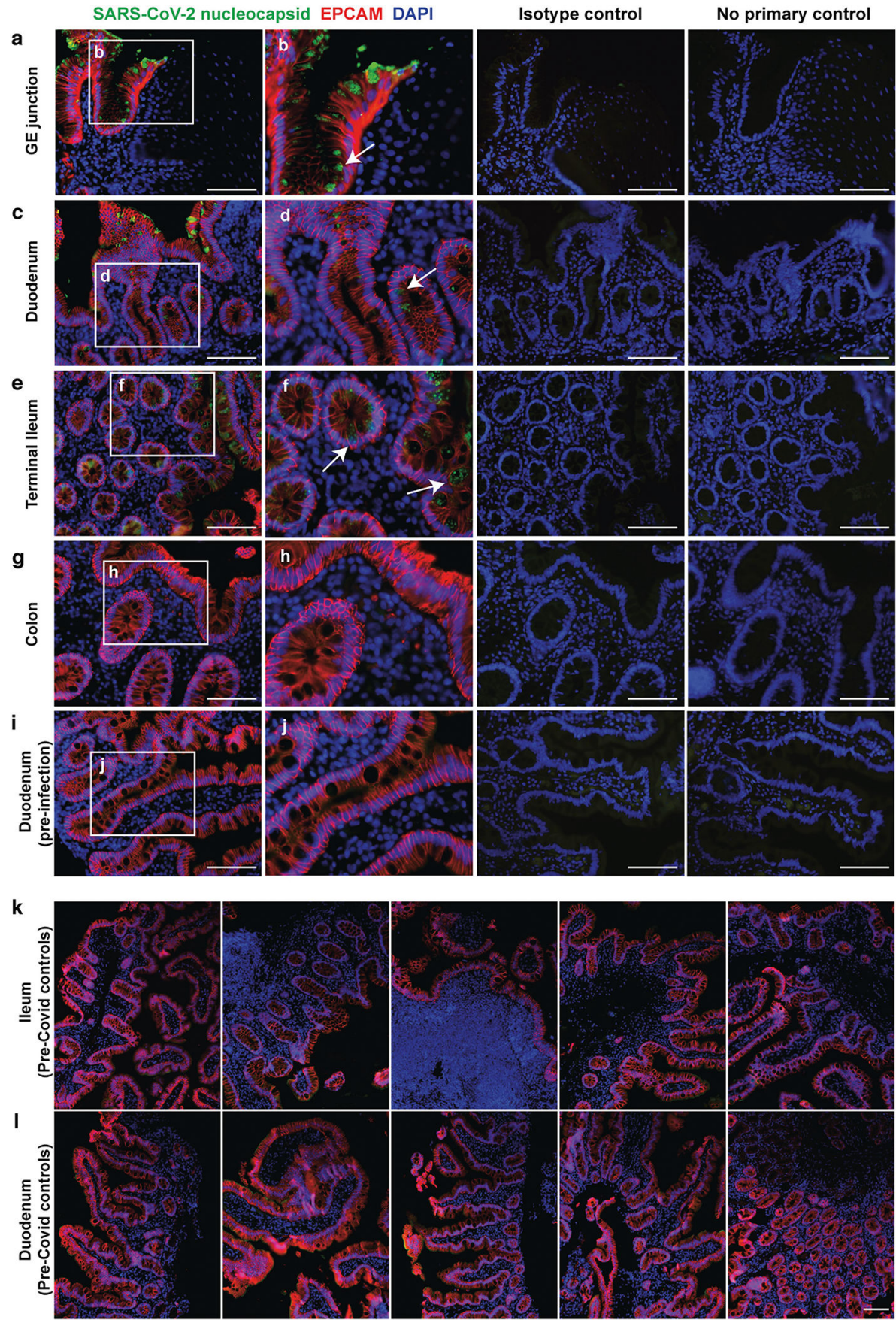

Extended Data Fig. 9 |. SARS-CoV-2 antigen in human enterocytes in the gastrointestinal tract at three months after COVID-19 diagnosis, and pre-COVID-19 control individuals without detectable SARS-CoV-2 antigen.

$\mathbf{a}-\mathbf{j}$, Immunofluorescence images of human gastrointestinal tissue are shown. Staining is for EPCAM (red), DAPI (blue) and SARS-CoV-2 nucleocapsid (green) Samples are derived from intestinal biopsies in the gastrointestinal tract as indicated. $\mathbf{a}-\mathbf{h}$, Biopsies from participant CGI-088 (Supplementary Table 7) taken $92 \mathrm{~d}$ after the onset of COVID-19 symptoms. i, j, Biopsy taken 27 months before the onset of COVID-19 symptoms from participant CGI-088. Arrows indicate enterocytes with detectable SARS-CoV-2 antigen. 
Isotype and no-primary controls for each tissue are shown in the right two columns. Scale bars, $100 \mu \mathrm{m}$. k, l, Immunofluorescence images of biopsy samples in the gastrointestinal tract (ileal (k) and duodenal (I)) obtained from ten preCOVID-19 control individuals between January 2018 and October 2019 are shown. Staining is for EPCAM (red), DAPI (blue) and SARS-CoV-2 nucleocapsid (green). Scale bars, $100 \mu \mathrm{m}$. All experiments were repeated independently at least twice with similar results.
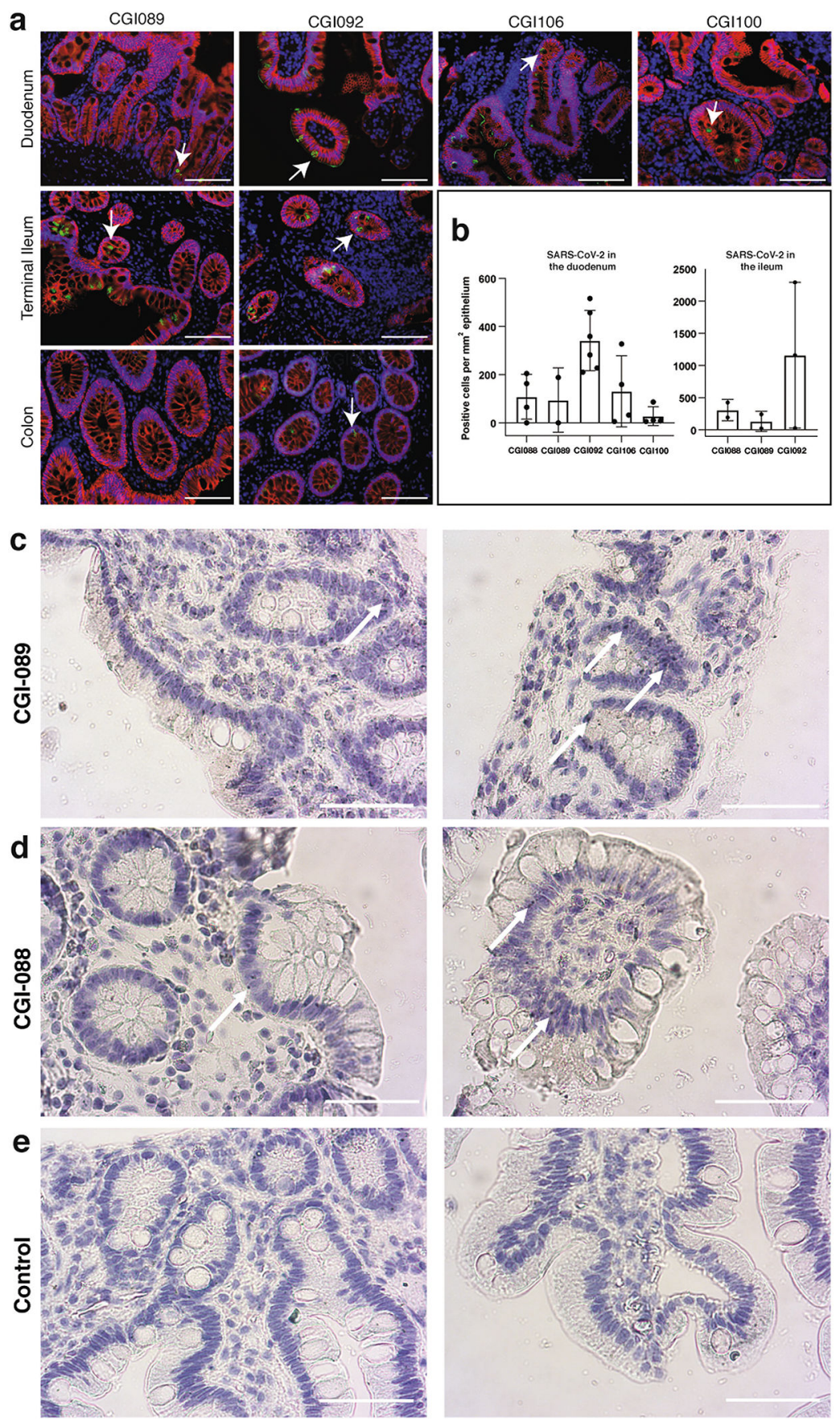

Extended Data Fig. 10 |. SARS-CoV-2 antigen and RNA is detectable in different intestinal segments in several individuals convalescent from COVID-19. 
a, Immunofluorescence images of biopsy samples in the gastrointestinal tract in different individuals are shown. Staining is for EPCAM (red), DAPI (blue) and SARS-CoV-2 nucleocapsid (green). Samples are derived from intestinal biopsies from four participants (CGI-089, CGI-092, CGI-100 and CGI-106) taken at least three months after COVID-19 infection. Arrows indicate enterocytes with detectable SARS-CoV-2 antigen. Scale bars, 100 $\mu \mathrm{m}$. The experiments were repeated independently at least twice with similar results. $\mathbf{b}$, Quantification of SARS-CoV-2-positive cells by immunofluorescence. The number of cells staining positive for the $\mathrm{N}$ protein of SARS-CoV-2 per $\mathrm{mm}^{2}$ of intestinal epithelium is shown. The graphs show biopsy samples from the indicated individuals of the duodenum (left) and terminal ileum (right). Black dots represent the number of available biopsy specimen for each individual from the respective intestinal segment (CGI-088, $n=4$ duodenal and $n=2$ ileal; CGI-089, $n=2$ duodenal and $n=2$ ileal; CGI-092, $n=6$ duodenal and $n=3$ ileal; CGI-106, $n=4$; CGI-100, $n=4$ ). Boxes represent median values and whiskers the $95 \%$ confidence interval. c, d, SARS-CoV-2 viral RNA was visualized in intestinal biopsies of participant CGI-089 (c) and CGI-088 (d) using in situ hybridization. SARS-CoV-2 genomic RNA (black) and haematoxylin and eosin staining by smFISHimmunohistochemistry technique in the duodenum (left) or terminal ileum (right). Arrows indicate enterocytes with detectable SARS-CoV-2 RNA. e, Pre-COVID-19 control individuals show no detectable SARS-CoV-2 viral RNA in duodenal (left) or ileal (right) biopsies. Scale bars, $100 \mu \mathrm{m}$. The experiments were repeated independently three times with similar results.

\section{Supplementary Material}

Refer to Web version on PubMed Central for supplementary material.

\section{Acknowledgements}

We thank all study participants who devoted time to our research; B. Coller and S. Schlesinger, the Rockefeller University Hospital Clinical Research Support Office and nursing staff; M. Okawa Frank and R. B. Darnell for SARS-CoV-2 saliva PCR testing; C. M. Rice and all members of the M.C.N. laboratory for helpful discussions; M. Jankovic for laboratory support; and J. Vielmetter and the Protein Expression Center in the Beckman Institute at Caltech for expression assistance. This work was supported by NIH grant P01-AI138398-S1 (M.C.N. and P.J.B.) and 2U19AI111825 (M.C.N.).; the Caltech Merkin Institute for Translational Research and P50 AI150464-13 (P.J.B.), George Mason University Fast Grant (D.F.R. and P.J.B.) and the European ATAC grant EC 101003650 (D.F.R.); R37-AI64003 to P.D.B.; R01AI78788 to T. Hatziioannou; NCI R01CA234614, NIAID 2R01 AI107301, NIDDK R01DK121072 and 1RO3DK117252 to R.E.S., NIH NIDDK R01 DK123749 01S1 to S.M. C.O.B. is supported by the HHMI Hanna Gray and Burroughs Wellcome PDEP fellowships. R.E.S. is an Irma Hirschl Trust Research Award Scholar. M.T. is supported by the Digestive Disease Research Foundation (DDRF). C.G. was supported by the Robert S. Wennett Post-Doctoral Fellowship, in part by the National Center for Advancing Translational Sciences (National Institutes of Health Clinical and Translational Science Award programme, grant UL1 TR001866), and by the Shapiro-Silverberg Fund for the Advancement of Translational Research. P.D.B. and M.C.N. are Howard Hughes Medical Institute Investigators.

\section{Data availability}

Data are provided in Supplementary Tables 1-8. The raw sequencing data and computer scripts associated with Fig. 2 have been deposited at https://github.com/stratust/igpipeline. This study also uses data from a DRYAD accession linked to ref. ${ }^{57}$ (https://doi.org/10.5061/ dryad.35ks2), the Protein Data Bank (accession codes 6VYB and 6NB6) and ref. ${ }^{53}$. 


\section{References}

1. Robbiani DF et al. Convergent antibody responses to SARS-CoV-2 in convalescent individuals. Nature 584, 437-442 (2020). [PubMed: 32555388]

2. Schäfer A et al. Antibody potency, effector function, and combinations in protection and therapy for SARS-CoV-2 infection in vivo. J. Exp. Med 218, e20201993 (2021). [PubMed: 33211088]

3. Carfì A, Bernabei R \& Landi F Persistent symptoms in patients after acute COVID-19. J. Am. Med. Assoc 324, 603-605 (2020).

4. del Rio C., Collins LF. \& Malani P. Long-term Health Consequences of COVID-19. JAMA 324, 1723-1724 (2020). [PubMed: 33031513]

5. Yang HS et al. SARS-CoV-2 antibody characterization in emergency department, hospitalized and convalescent patients by two semi-quantitative immunoassays. Clin. Chim. Acta 509, 117-125 (2020). [PubMed: 32505774]

6. Diagnostics Roche. Roche Diagnostics Elecsys Anti-SARS-CoV-2, https://www.fda.gov/media/ 137605 (2020).

7. Muecksch F et al. Longitudinal analysis of serology and neutralizing antibody levels in COVID19 convalescents. J. Infect. Dis jiaa659 (2020).

8. Schmidt F et al. Measuring SARS-CoV-2 neutralizing antibody activity using pseudotyped and chimeric viruses. J. Exp. Med 217, e20201181 (2020). [PubMed: 32692348]

9. Wajnberg A et al. Robust neutralizing antibodies to SARS-CoV-2 infection persist for months. Science 370, 1227-1230 (2020). [PubMed: 33115920]

10. Seow $\mathbf{J}$ et al. Longitudinal observation and decline of neutralizing antibody responses in the three months following SARS-CoV-2 infection in humans. Nat. Microbiol 5, 1598-1607 (2020). [PubMed: 33106674]

11. Brouwer PJM et al. Potent neutralizing antibodies from COVID-19 patients define multiple targets of vulnerability. Science 369, 643-650 (2020). [PubMed: 32540902]

12. Cao $\mathrm{Y}$ et al. Potent neutralizing antibodies against SARS-CoV-2 identified by high-throughput single-cell sequencing of convalescent patients' B cells. Cell 182, 73-84 (2020). [PubMed: 32425270]

13. Ju B et al. Human neutralizing antibodies elicited by SARS-CoV-2 infection. Nature 584, 115-119 (2020). [PubMed: 32454513]

14. Kreer $\mathrm{C}$ et al. Longitudinal isolation of potent near-germline SARS-CoV-2-neutralizing antibodies from COVID-19 patients. Cell 182, 843-854 (2020). [PubMed: 32673567]

15. Seydoux E et al. Analysis of a SARS-CoV-2-infected individual reveals development of potent neutralizing antibodies with limited somatic mutation. Immunity 53, 98-105 (2020). [PubMed: 32561270]

16. Yuan M et al. Structural basis of a shared antibody response to SARS-CoV-2. Science 369, 11191123 (2020). [PubMed: 32661058]

17. Weisblum $\mathrm{Y}$ et al. Escape from neutralizing antibodies by SARS-CoV-2 spike protein variants. eLife 9, e61312 (2020). [PubMed: 33112236]

18. Barnes $\mathrm{CO}$ et al. SARS-CoV-2 neutralizing antibody structures inform therapeutic strategies. Nature 588, 682-687 (2020). [PubMed: 33045718]

19. Tortorici MA et al. Ultrapotent human antibodies protect against SARS-CoV-2 challenge via multiple mechanisms. Science 370, 950-957 (2020). [PubMed: 32972994]

20. Baum A et al. Antibody cocktail to SARS-CoV-2 spike protein prevents rapid mutational escape seen with individual antibodies. Science 369, 1014-1018 (2020). [PubMed: 32540904]

21. Li Q et al. The impact of mutations in SARS-CoV-2 spike on viral infectivity and antigenicity. Cell 182, 1284-1294 (2020). [PubMed: 32730807]

22. Lamers MM et al. SARS-CoV-2 productively infects human gut enterocytes. Science $369,50-54$ (2020). [PubMed: 32358202]

23. Livanos AE et al. Gastrointestinal involvement attenuates COVID-19 severity and mortality. Preprint at 10.1101/2020.09.07.20187666 (2020). 
24. Qian Q et al. Direct evidence of active SARS-CoV-2 replication in the intestine. Clin. Infect. Dis ciaa925 (2020).

25. Han $\mathrm{Y}$ et al. Identification of SARS-CoV-2 inhibitors using lung and colonic organoids. Nature 589, 270-275 (2021). [PubMed: 33116299]

26. Morone $\mathrm{G}$ et al. Incidence and persistence of viral shedding in COVID-19 post-acute patients with negativized pharyngeal swab: a systematic review. Front. Med 7, 562 (2020).

27. Park SK et al. Detection of SARS-CoV-2 in fecal samples from patients with asymptomatic and mild COVID-19 in Korea. Clin. Gastroenterol. Hepatol 10.1016/j.cgh.2020.06.005 (2020).

28. Wölfel R et al. Virological assessment of hospitalized patients with COVID-2019. Nature 581, 465-469 (2020). [PubMed: 32235945]

29. Beaudoin-Bussières $\mathrm{G}$ et al. Decline of humoral responses against SARS-CoV-2 spike in convalescent individuals. MBio 11, e02590-20398 (2020).

30. Crawford KHD et al. Dynamics of neutralizing antibody titers in the months after SARS-CoV-2 infection. J. Infect. Dis jiaa618 (2020).

31. Iyer AS et al. Persistence and decay of human antibody responses to the receptor binding domain of SARS-CoV-2 spike protein in COVID-19 patients. Sci. Immunol 5, eabe0367-0313 (2020). [PubMed: 33033172]

32. Wang $\mathrm{K}$ et al. Longitudinal dynamics of the neutralizing antibody response to SARS-CoV-2 infection. Clin. Infect. Dis 579, 270-279 (2020).

33. Ripperger TJ et al. Orthogonal SARS-CoV-2 serological assays enable surveillance of lowprevalence communities and reveal durable humoral immunity. Immunity 53, 925-933.e4 (2020). [PubMed: 33129373]

34. Dan JM et al. Immunological memory to SARS-CoV-2 assessed for up to 8 months after infection. Science 10.1126/science.abf4063 (2021).

35. Rodda LB et al. Functional SARS-CoV-2-specific immune memory persists after mild COVID-19. Cell 184, 169-183 (2021). [PubMed: 33296701]

36. Secchi M et al. COVID-19 survival associates with the immunoglobulin response to the SARSCoV-2 spike receptor binding domain. J. Clin. Invest 130, 6366-6378 (2020). [PubMed: 32991329]

37. Liu L et al. Potent neutralizing antibodies against multiple epitopes on SARS-CoV-2 spike. Nature 584, 450-456 (2020). [PubMed: 32698192]

38. Zost SJ et al. Potently neutralizing and protective human antibodies against SARS-CoV-2. Nature 584, 443-449 (2020). [PubMed: 32668443]

39. Nielsen SCA et al. Human B cell clonal expansion and convergent antibody responses to SARSCoV-2. Cell Host Microb 28, 516-525 (2020).

40. Kaneko $\mathrm{N}$ et al. Loss of Bcl-6-expressing T follicular helper cells and germinal centers in COVID-19. Cell 183, 143-157 (2020). [PubMed: 32877699]

41. Victora GD \& Nussenzweig MC Germinal centers. Annu. Rev. Immunol 30, 429-457 (2012). [PubMed: 22224772]

42. Sokal A et al. Maturation and persistence of the anti-SARS-CoV-2 memory B cell response. Preprint at 10.1101/2020.11.17.385252 (2020).

43. Mahler DA \& Wells CK Evaluation of clinical methods for rating dyspnea. Chest 93, 580-586 (1988). [PubMed: 3342669]

44. Pujadas E et al. SARS-CoV-2 viral load predicts COVID-19 mortality. Lancet Respir. Med 8, e70 (2020). [PubMed: 32771081]

45. Chomczynski P \& Sacchi N Single-step method of RNA isolation by acid guanidinium thiocyanate-phenol-chloroform extraction. Anal. Biochem 162, 156-159 (1987). [PubMed: 2440339]

46. DeAngelis MM, Wang DG \& Hawkins TL Solid-phase reversible immobilization for the isolation of PCR products. Nucleic Acids Res. 23, 4742-4743 (1995). [PubMed: 8524672]

47. Grifoni A. et al.. Targets of $\mathrm{T}$ cell responses to SARS-CoV-2 coronavirus in humans with COVID-19 disease and unexposed individuals. Cell 181, 1489-1501 (2020). [PubMed: 32473127] 
48. Amanat $\mathrm{F}$ et al. A serological assay to detect SARS-CoV-2 seroconversion in humans. Nat. Med 26, 1033-1036 (2020). [PubMed: 32398876]

49. Barnes CO et al. Structures of human antibodies bound to SARS-CoV-2 spike reveal common epitopes and recurrent features of antibodies. Cell 182, 828-842 (2020). [PubMed: 32645326]

50. Weisblum $\mathrm{Y}$ et al. Escape from neutralizing antibodies by SARS-CoV-2 spike protein variants. eLife 9, e61312 (2020). [PubMed: 33112236]

51. Van Gassen $\mathrm{S}$ et al. FlowSOM: using self-organizing maps for visualization and interpretation of cytometry data. Cytometry A 87, 636-645 (2015). [PubMed: 25573116]

52. Gupta NT et al. Change-O: a toolkit for analyzing large-scale B cell immunoglobulin repertoire sequencing data. Bioinformatics 31, 3356-3358 (2015). [PubMed: 26069265]

53. Soto $\mathrm{C}$ et al. High frequency of shared clonotypes in human $\mathrm{B}$ cell receptor repertoires. Nature 566, 398-402 (2019). [PubMed: 30760926]

54. Guo Y, Chen K, Kwong PD, Shapiro L \& Sheng Z cAb-Rep: a database of curated antibody repertoires for exploring antibody diversity and predicting antibody prevalence. Front. Immunol 10, 2365 (2019). [PubMed: 31649674]

55. Kyte J \& Doolittle RF A simple method for displaying the hydropathic character of a protein. J. Mol. Biol 157, 105-132 (1982). [PubMed: 7108955]

56. Guy HR Amino acid side-chain partition energies and distribution of residues in soluble proteins. Biophys. J 47, 61-70 (1985). [PubMed: 3978191]

57. DeWitt WS et al. A public database of memory and naive B-cell receptor sequences. PLoS ONE 11, e0160853 (2016). [PubMed: 27513338]

58. Spiegel $\mathrm{M}$ et al. Inhibition of beta interferon induction by severe acute respiratory syndrome coronavirus suggests a two-step model for activation of interferon regulatory factor 3. J. Virol 79, 2079-2086 (2005). [PubMed: 15681410] 

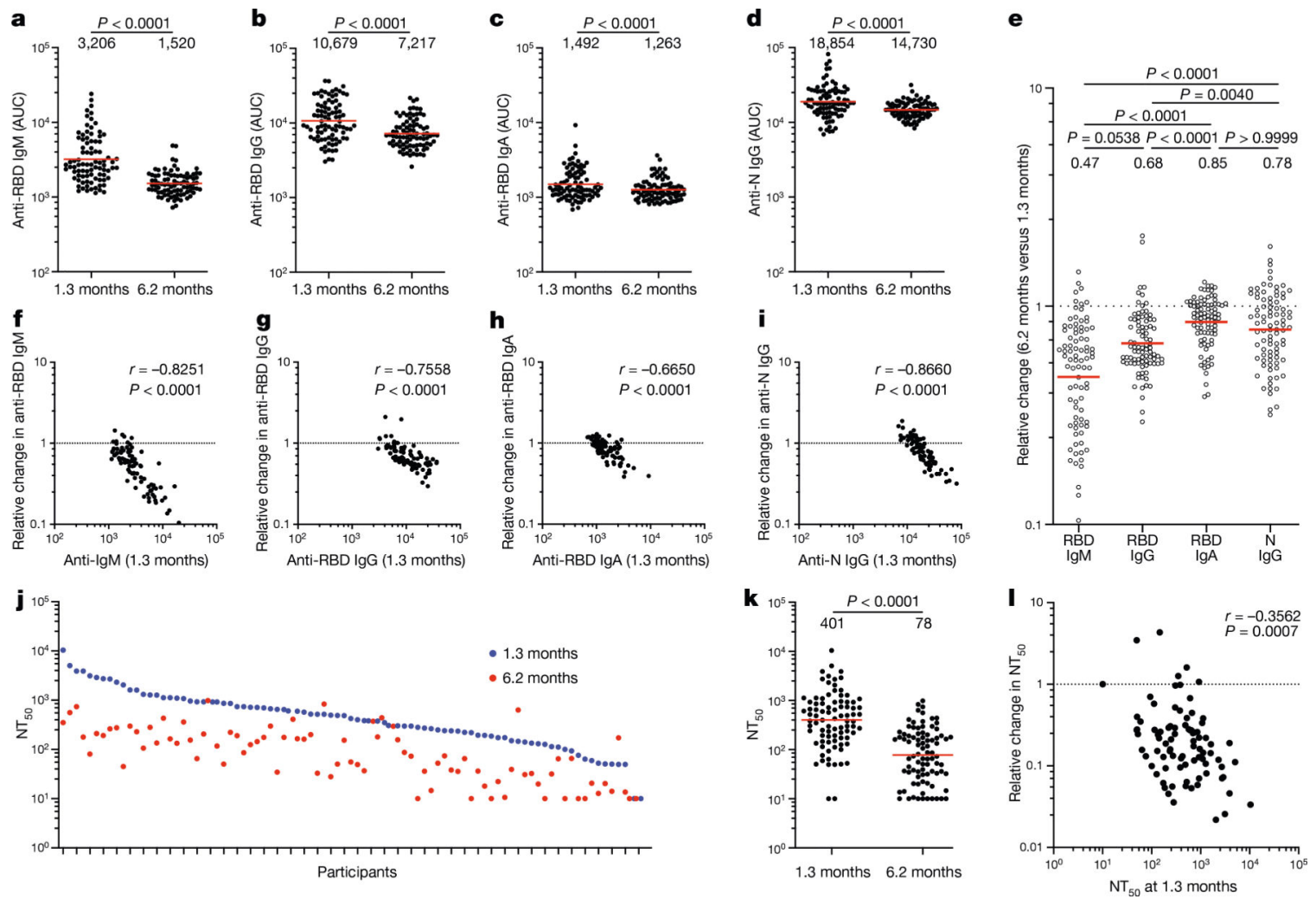

Fig. 1 |. Plasma antibody dynamics against SARS-CoV-2.

a-d, Results of ELISAs measuring plasma reactivity to $\operatorname{RBD}(\mathbf{a}, \mathbf{b}, \mathbf{c})$ and $\mathrm{N}$ protein $(\mathbf{d})$ at the initial 1.3- and 6.2-month follow-up visit, respectively. a, Anti-RBD IgM. b, Anti-RBD IgG. c, Anti-RBD IgA d, Anti-N IgG. The normalized area under the curve (AUC) values for 87 individuals are shown in a-d for both time points. Positive and negative controls were included for validation ${ }^{1}$. e, Relative change in plasma antibody levels between 1.3 and 6.2 months for anti-RBD IgM, IgG, IgA and anti-N IgG in all 87 individuals. $\mathbf{f}-\mathbf{i}$, Relative change in antibody levels between 1.3 and 6.2 months plotted against the corresponding antibody levels at 1.3 months. f, Anti-RBD IgM. $r=-0.83, P<0.0001$. g, Anti-RBD IgG. $r$ $=-0.76,1.3$ months 6.2 months $100101102103104105 \mathrm{NT}_{50}$ at 1.3 months $P<0.0001$. h, Anti-RBD IgA. $r=-0.67, P<0.0001$. i, Anti-N IgG. $r=-0.87, P<0.0001$. j, Ranked average $\mathrm{NT}_{50}$ at 1.3 months (blue) and 6.2 months (red) for the 87 individuals studied. $\mathbf{k}$, Graph shows $\mathrm{NT}_{50}$ for plasma from all 87 individuals collected at 1.3 and 6.2 months. $P<$ 0.0001 . I, Relative change in plasma neutralizing titres between 1.3 and 6.2 months plotted against the corresponding titres at 1.3 months. For $\mathbf{a}-\mathbf{e}, \mathbf{k}$ plotted values and horizontal bars indicate geometric mean. Statistical significance was determined using two-tailed Wilcoxon matched-pairs signed-rank test in $\mathbf{a}-\mathbf{d}, \mathbf{k}$, and Friedman with Dunn's multiple comparison test in $\mathbf{e}$. The $r$ and $P$ values in $\mathbf{f}-\mathbf{i}, \mathbf{I}$ were determined by two-tailed Spearman's correlations. 

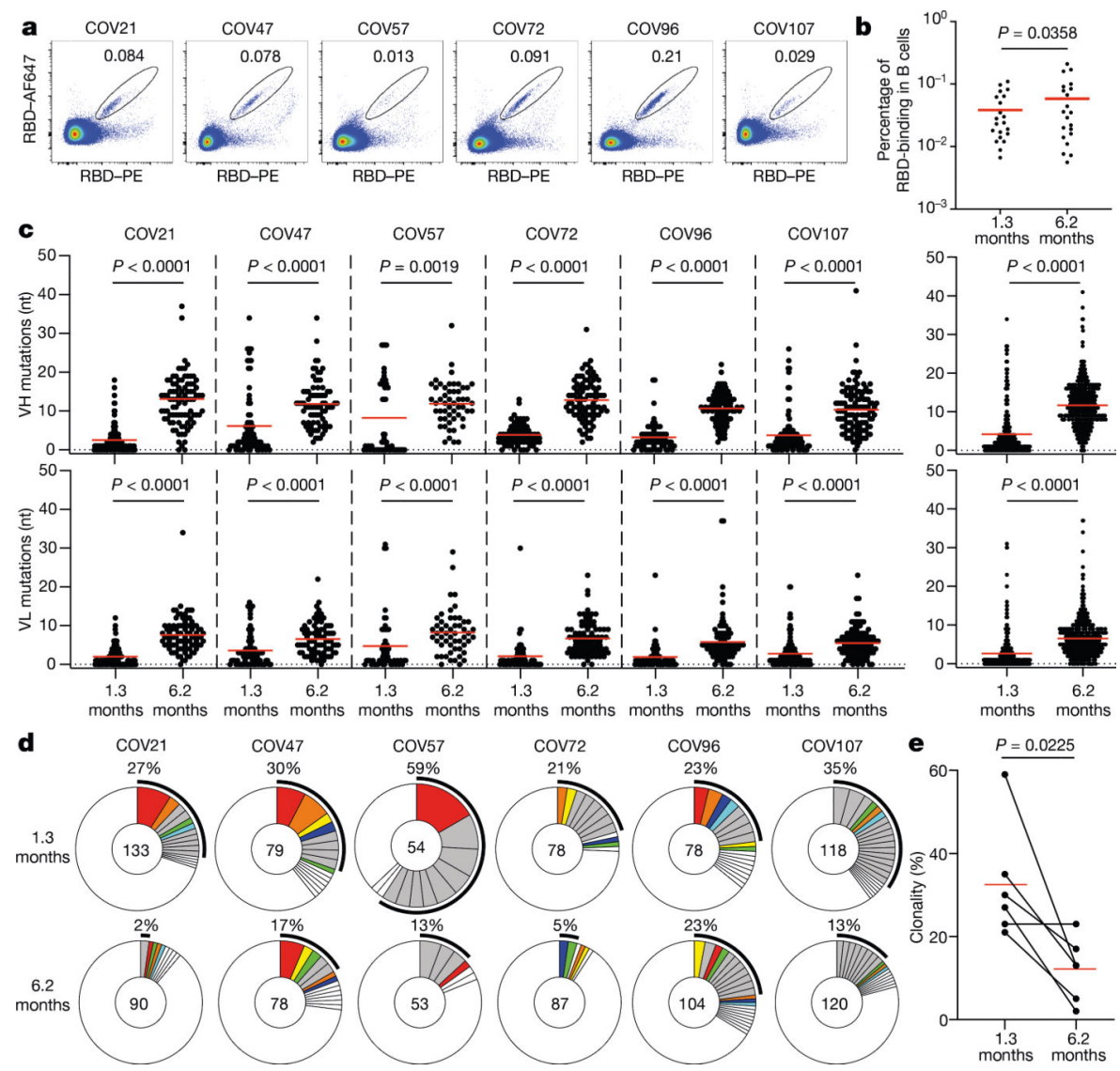

Fig. 2 |. Sequences of anti-SARS-CoV-2 RBD antibodies.

a, Representative flow cytometry plots showing dual AlexaFluor-647-RBD- and PE-RBDbinding B cells for six study individuals (designated COV21, COV47, COV57, COV72, COV96 and COV107) (the gating strategy is shown in Extended Data Fig. 5). The percentage of antigen-specific B cells is indicated. $\mathbf{b}$, As in $\mathbf{a}$. Graph summarizes the percentage of RBD-binding memory B cells in samples obtained at 1.3 and 6.2 months from 21 randomly selected individuals. Red horizontal bars indicate geometric mean values. Statistical significance was determined using two-tailed Wilcoxon matched-pairs signedrank test. c, Number of somatic nucleotide mutations in the IGVH (top) and IGVL (bottom) genes in antibodies obtained after 1.3 or 6.2 months from the indicated individual (left) or all six donors (right). Statistical significance was determined using two-tailed Mann-Whitney $U$-tests. Horizontal bars indicate median values. d, Pie charts show the distribution of antibody sequences from six individuals after $1.3^{1}$ (top) or 6.2 months (bottom). The number in the inner circle indicates the number of sequences analysed for the individual denoted above the circle. Pie-slice size is proportional to the number of clonally related sequences. The black outline indicates the frequency of clonally expanded sequences detected in each participant. Coloured slices indicate persisting clones (same IGHV and IGLV genes and highly similar CDR3) found at both time points in the same participant. Grey slices indicate clones unique to the time point. White slices indicate singlets found at both time points, and the remaining white area indicates sequences that were isolated once. e, Graph shows 
relative clonality at both time points for all six donors. Red horizontal bars indicate mean values. Statistical significance was determined using two-tailed $t$-test. 

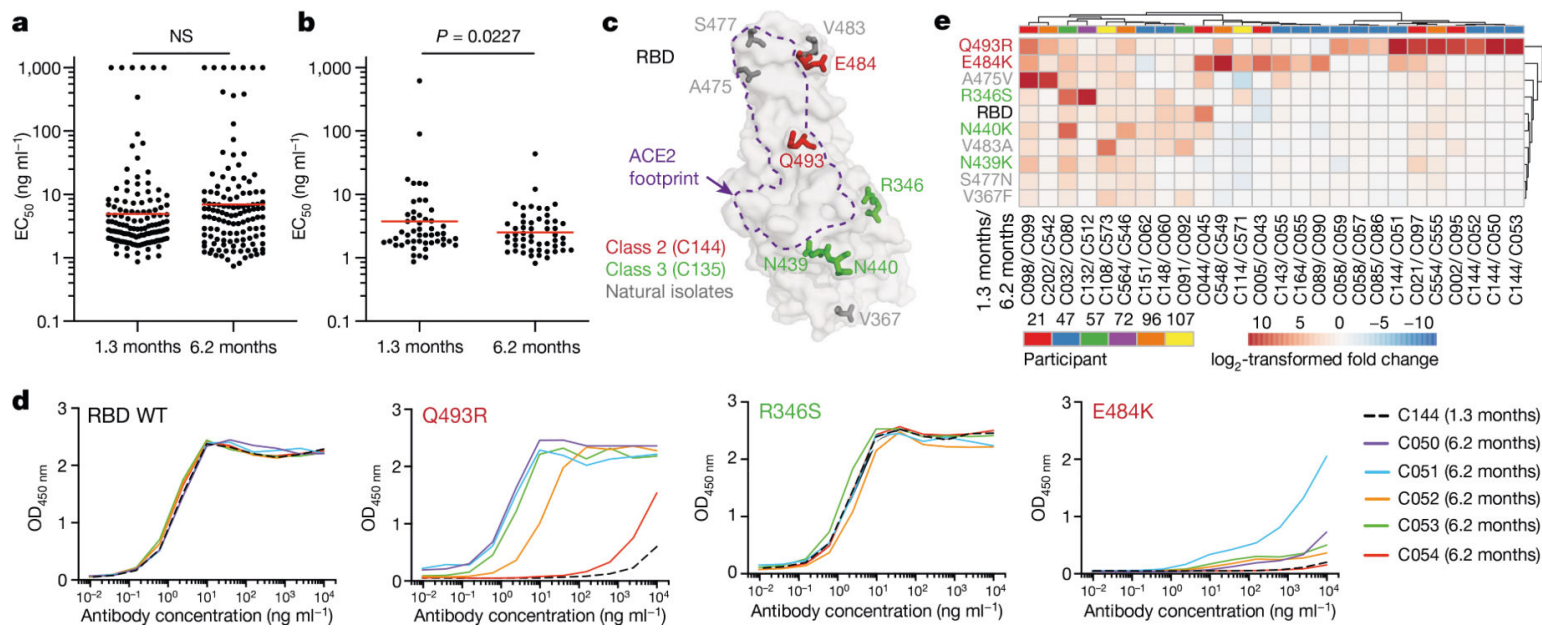

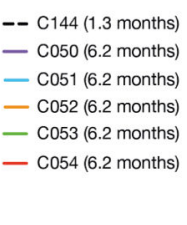

Fig. 3 |. Reactivity of anti-SARS-CoV-2 RBD monoclonal antibodies.

a, Graph shows anti-SARS-CoV-2 RBD antibody reactivity. ELISA EC 50 values for all antibodies measured at 1.3 months ${ }^{1}$, and 122 selected monoclonal antibodies measured at 6.2 months. Horizontal bars indicate geometric mean. Statistical significance was determined using two-tailed Mann-Whitney $U$-test. b, $\mathrm{EC}_{50}$ values for all 52 antibodies that appear at 1.3 and 6.2 months. Average of two or more experiments. Horizontal bars indicate geometric mean. Statistical significance was determined using two-tailed Wilcoxon matched-pairs signed-rank test. c, Surface representation of the RBD with the ACE2-binding footprint indicated as a dotted line and selected residues found in circulating strains (grey) and residues that mediate resistance to class-2 (red) (C144) and -3 (green) (C135) antibodies highlighted as sticks. d, Graphs show ELISA binding curves for C144 (black dashed line) and its clonal relatives obtained after 6.2 months (C050, C051, C052, C053 and C054) (solid lines) binding to wild-type (WT), Q493R, R346S and E484K RBDs. e. Heat map shows $\log _{2}$-transformed relative fold change in $\mathrm{EC}_{50}$ against indicated $\mathrm{RBD}$ mutants for 26 antibody clonal pairs obtained at 1.3 and 6.2 months with the most pronounced changes in reactivity. The participant of origin for each antibody pair is indicated above. All experiments were performed at least twice. 

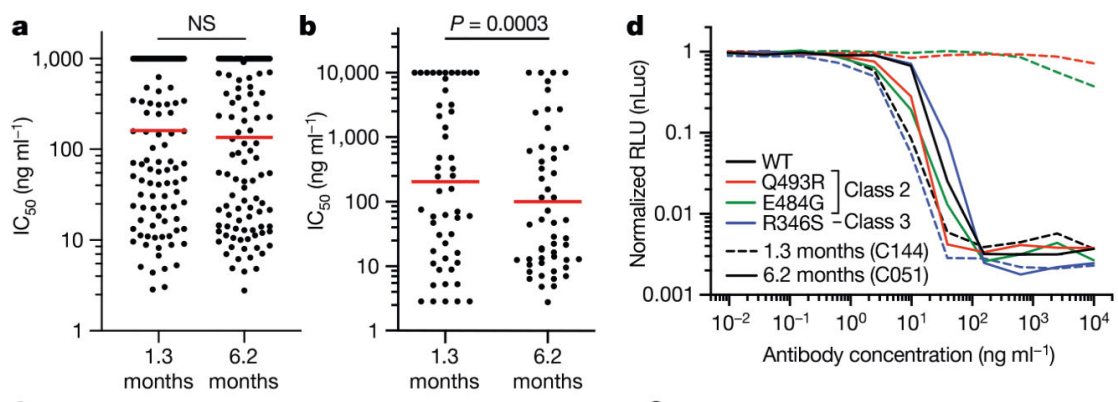

c

\begin{tabular}{cccccc}
\multirow{2}{*}{$\begin{array}{c}\text { Antibody } \\
\text { ID }\end{array}$} & Obtained at & \multicolumn{4}{c}{$\mathrm{IC}_{50}\left(\mathrm{ng} \mathrm{m}^{-1}\right)$} \\
\cline { 3 - 6 } & $\mathrm{WT}$ & $\mathrm{Q} 493 \mathrm{R}$ & $\mathrm{E} 484 \mathrm{G}$ & $\mathrm{R} 346 \mathrm{~S}$ \\
\hline C144 & 1.3 months & 2.73 & $>10,000$ & $\mathbf{4 , 1 1 4 . 0 7}$ & 1.78 \\
C051 & 6.2 months & 11.99 & $\mathbf{4 . 6 8}$ & $\mathbf{3 . 1 0}$ & 13.56 \\
\hline C548 & 1.3 months & 19.13 & $>10,000$ & $\mathbf{1 , 5 9 1 . 7 7}$ & 10.84 \\
C549 & 6.2 months & 10.95 & $\mathbf{2 . 3 5}$ & $\mathbf{2 . 2 9}$ & 8.33 \\
\hline C032 & 1.3 months & 100.69 & 62.42 & 45.68 & $>\mathbf{1 0 , 0 0 0}$ \\
C080 & 6.2 months & 21.52 & 21.25 & 6.00 & $\mathbf{5 . 3 1}$ \\
\hline C132 & 1.3 months & $1,024.35$ & 796.48 & $1,406.57$ & $>\mathbf{1 0 , 0 0 0}$ \\
C512 & 6.2 months & 21.55 & 20.78 & 12.17 & $\mathbf{4 , 0 2 2 . 0 2}$ \\
\hline C164 & 1.3 months & 87.89 & 139.53 & 369.18 & 57.24 \\
C143 & 1.3 months & 191.23 & $1,576.12$ & $4,402.39$ & 224.99 \\
C055 & 6.2 months & 4.98 & 6.52 & 44.78 & 4.03 \\
\hline & & & & &
\end{tabular}

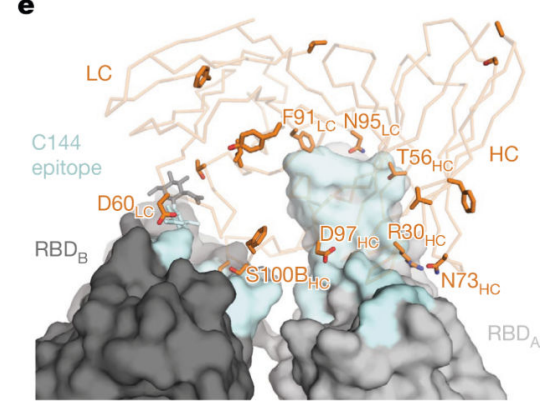

Fig. 4 |. Neutralizing activity of anti-SARS-CoV-2 RBD monoclonal antibodies.

a, SARS-CoV-2 pseudovirus neutralization assay. $\mathrm{IC}_{50}$ values for all antibodies measured at 1.3 months $^{1}$, and 122 selected antibodies measured at 6.2 months. Antibodies with $\mathrm{IC}_{50}$ values above $1 \mu \mathrm{g} \mathrm{ml}^{-1}$ were plotted at $1 \mu \mathrm{g} \mathrm{m}{ }^{-1}$. Mean of two independent experiments. Red bar indicates geometric mean. Statistical significance was determined using two-tailed Mann-Whitney $U$-test. b, $\mathrm{IC}_{50}$ values for 52 antibodies appearing at 1.3 and 6.2 months. Red bar indicates geometric mean. Statistical significance was determined using two-tailed Wilcoxon matched-pairs signed-rank test. c, $\mathrm{IC}_{50}$ values for 5 pairs of monoclonal antibody clonal relatives obtained after 1.3 or 6.2 months for neutralization of wild-type and mutant SARS-CoV-2 pseudovirus. Antibody identifiers of the 1.3-month-6.2-month monoclonal antibody pairs as indicated. Bold styling denotes antibody pairs with substantial increase in neutralizing activity after 6.2 months. d, Graph shows the normalized relative luminescence units (RLU) for cell lysates of 293T cells expressing ACE2, $48 \mathrm{~h}$ after infection with SARSCoV-2 pseudovirus containing wild-type RBD or one of three mutant RBDs (Q493R, E484G and R346S) in the presence of increasing concentrations of one of two monoclonal antibodies C144 (1.3 months) (dashed lines) or C051 (6.2 months) (solid lines). Experiments were performed at least twice. e, C051 binding model. Surface representation of two adjacent 'down' $\mathrm{RBDs}\left(\mathrm{RBD}_{\mathrm{A}}\right.$ and $\left.\mathrm{RBD}_{\mathrm{B}}\right)$ on a spike trimer, with the $\mathrm{C} 144$ epitope on the RBDs highlighted in cyan and positions of amino acid mutations that accumulated in C051 compared to the parent antibody $\mathrm{C} 144$ highlighted as stick side chains on a $\mathrm{Ca}$ atom representation of $\mathrm{C} 051 \mathrm{~V}_{\mathrm{H}} \mathrm{V}_{\mathrm{L}}$ binding to adjacent RBDs. The C051 interaction with two RBDs was modelled on the basis of a cryo-electron microscopy structure of C144 Fab bound to spike trimer ${ }^{18}$. HC, heavy chain; LC, light chain. 


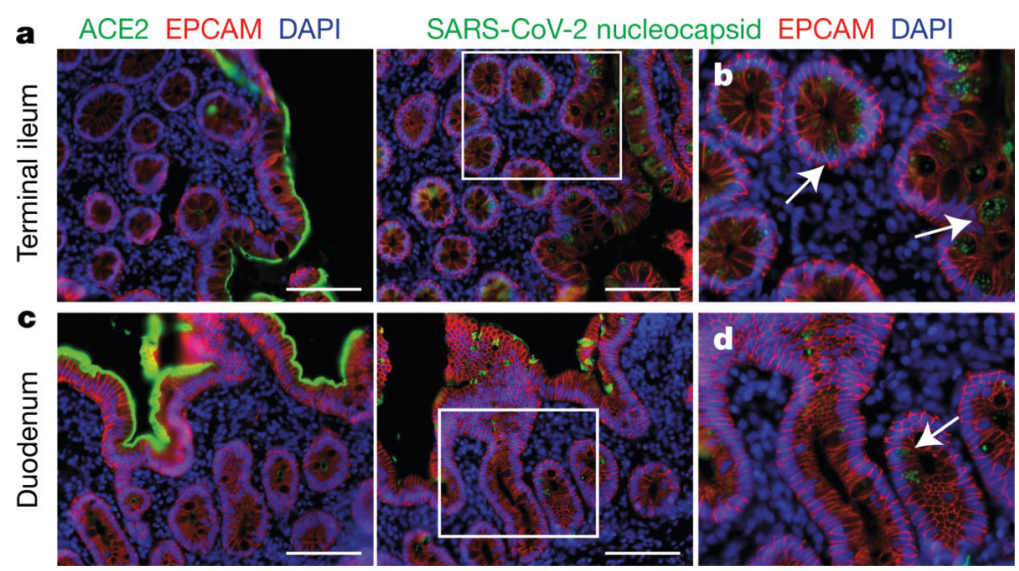

Fig. 5 |. Immunofluorescence imaging of intestinal biopsies.

a, Immunofluorescence images of human enterocytes stained for EPCAM (red), DAPI (blue) and either ACE2 (green in a, c) or SARS-CoV-2 N (green in b, d) in intestinal biopsies taken $92 \mathrm{~d}$ after onset of COVID-19 symptoms in participant CGI-088, in the terminal ileum $(\mathbf{a}, \mathbf{b})$ or duodenum $(\mathbf{c}, \mathbf{d})$. Regions in white boxes in the right panels of $\mathbf{a}, \mathbf{c}$ are shown expanded in b, d, respectively. Arrows indicate enterocytes with detectable SARS-CoV-2 antigen. Scale bars, $100 \mu \mathrm{m}$. The experiments were repeated independently at least twice with similar results. 